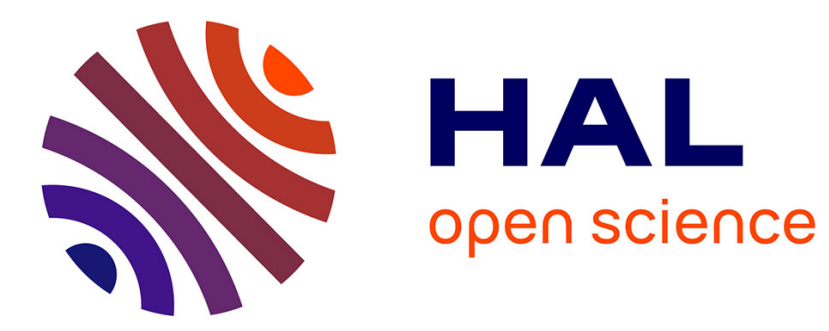

\title{
Multiplicity-Induced-Dominancy in parametric second-order delay differential equations: Analysis and application in control design
}

Islam Boussaada, Silviu-Iulian Niculescu, Ali El Ati, Redamy Pérez-Ramos, Karim Liviu Trabelsi

\section{To cite this version:}

Islam Boussaada, Silviu-Iulian Niculescu, Ali El Ati, Redamy Pérez-Ramos, Karim Liviu Trabelsi. Multiplicity-Induced-Dominancy in parametric second-order delay differential equations: Analysis and application in control design. ESAIM: Control, Optimisation and Calculus of Variations, inPress, $10.1051 / \mathrm{cocv} / 2019073$. hal-02421253

\section{HAL Id: hal-02421253 \\ https://hal.science/hal-02421253}

Submitted on 20 Dec 2019

HAL is a multi-disciplinary open access archive for the deposit and dissemination of scientific research documents, whether they are published or not. The documents may come from teaching and research institutions in France or abroad, or from public or private research centers.
L'archive ouverte pluridisciplinaire HAL, est destinée au dépôt et à la diffusion de documents scientifiques de niveau recherche, publiés ou non, émanant des établissements d'enseignement et de recherche français ou étrangers, des laboratoires publics ou privés. 


\title{
MULTIPLICITY-INDUCED-DOMINANCY IN PARAMETRIC SECOND-ORDER DELAY DIFFERENTIAL EQUATIONS: ANALYSIS AND APPLICATION IN CONTROL DESIGN
}

\author{
Islam BoussaAda $^{1}$, Silviu-Iulian Niculescu ${ }^{2}$, Ali El-Ati ${ }^{3}$, Redamy Pérez-Ramos ${ }^{4}$ \\ AND KARIM TRABELSI ${ }^{5}$
}

\begin{abstract}
This work revisits recent results on maximal multiplicity induced-dominancy for spectral values in reduced-order time-delay Systems and extends it to the general class of second-order retarded differential equations. A parametric multiplicity-induced-dominancy property is characterized, allowing to a delayed stabilizing design with reduced complexity. As a matter of fact, the approach is merely a delayed-output-feedback where the candidates' delays and gains result from the manifold defining the maximal multiplicity of a real spectral value, then, the dominancy is shown using the argument principle. Sensitivity of the control design with respect to the parameters uncertainties/variation is discussed. Various reduced order examples illustrate the applicative perspectives of the approach.

Résumé. Ce travail revisite les résultats récents sur la dominance induite par la multiplicité maximale des valeurs spectrales dans les systèmes à retard d'ordre réduit et l'étend à la classe la plus générale générale des équations différentielles de second ordre impliquant un retard. La propriété de dominance induite par la multiplicité est paramétriquement caractérisée, permettant la conception de loi de commande retardée, stabilisante et à complexité réduite. En effet, l'approche consiste simplement en un retour de sortie retardé dans lequel les retards et les gains des candidats résultent de la variété définissant la multiplicité maximale d'une valeur spectrale réelle, puis la dominance est montrée en utilisant le principe de l'argument. La sensibilité de la conception de la commande aux paramètres incertains/variant est discutée. Divers exemples d'ordre réduit illustrent les perspectives applicatives de l'approche.
\end{abstract}

1991 Mathematics Subject Classification. 4C60, 34K06, 35B35, 70J25.

$\cdots$

\footnotetext{
${ }^{1}$ The content of this paper was partially presented in The 23rd International Symposium on Mathematical Theory of Networks and Systems July 16-20, 2018. Hong Kong

Keywords and phrases: Time-delay systems, stability and stabilization, exponential decay, pole-placement, control design

${ }^{1}$ Inria Saclay, DISCO team \& L2S, Université Paris Saclay, CNRS-CentraleSupélec-Université Paris Sud, 3 rue Joliot-Curie, 91192 Gif-sur-Yvette cedex, France \& DRII-IPSA, Bis, 63 Boulevard de Brandebourg, 94200 Ivry-sur-Seine France

Islam.Boussaada@12s.centralesupelec.fr

${ }^{2}$ L2S, Université Paris Saclay, CNRS-CentraleSupélec-Université Paris Sud, 3 rue Joliot-Curie, 91192 Gif-sur-Yvette cedex, France \& Inria Saclay, DISCO team

Silviu.Niculescu@12s.centralesupelec.fr

${ }^{3}$ DRII-IPSA, Bis, 63 Boulevard de Brandebourg, 94200 Ivry-sur-Seine France.

Ali.El-Ati@ipsa.fr

${ }^{4}$ DRII-IPSA, Bis, 63 Boulevard de Brandebourg, 94200 Ivry-sur-Seine France.

Redamy.Perez-Ramos@ipsa.fr

${ }^{5}$ DRII-IPSA, Bis, 63 Boulevard de Brandebourg, 94200 Ivry-sur-Seine France.
}

Karim.Trabelsi@ipsa.fr 
TITLE WILL BE SET BY THE PUBLISHER

\section{INTRODUCTION}

It is commonly accepted that time-delay induces desynchronizing and/or destabilizing effects on the dynamics. However, new theoretical developments in the control of finite-dimensional dynamical systems suggest the use of delays in the control laws for stabilization purposes. The idea of exploiting the delay effect in controllers design was first introduced in [55] where it is shown that the conventional proportional controller equipped with an appropriate time-delay performs an averaged derivative action and thus can replace the proportional-derivative controller. Furthermore, it was stressed in [40] that time-delay has a stabilizing effect in the control design. Indeed, the closed-loop stability is guaranteed precisely by the existence of the delay. In the context of mechanical engineering problems, the effect of time-delay was emphasized in [54] where concrete applications are studied, such as the machine tool vibrations and some particular robotic systems.

This work focuses on the effect of multiplicity of spectral values on the exponential stability of the generic second-order retarded differential equation. The investigation of conditions on the equation parameters that guarantee the exponential stability of solutions is a question of ongoing interest, see for instance [19,28]. An efficient way to study a solution's stability is the frequency domain approach since in the Laplace domain, where a number of effective methods have been proposed, the stability analysis amounts to studying the distribution of the characteristic quasipolynomial function's roots, see for instance $[3,4,17,18,20,23,34,42,53,54,58,59]$.

It is worth noting that the rightmost root for a quasipolynomial function corresponding to stable time-delay systems is actually the exponential decay rate of its time-domain solution, see for instance [37] for an estimate of the decay rate for stable linear delay systems. To the best of the authors' knowledge, the first time an analytical proof of the dominancy of a spectral value for the scalar equation with a single delay was presented in [22]. The dominancy property is further explored and analytically shown in scalar delay equations in [14], then in second-order systems controlled by a delayed proportional is proposed in $[11,13]$ where its applicability in damping active vibrations for a piezo-actuated beam is proved. An extension to the delayed proportional-derivative controller case is studied in $[10,12]$ where the dominancy property is parametrically characterized and proven using the argument principle.

The strategy of using roots assignment for controller-design for time-delay systems is not new. As a matter of fact, in [30] a feedback law yields a finite spectrum of the closed-loop system located at an arbitrarily preassigned set of points in the complex plane. In the case of systems with delays in control only, a necessary and sufficient condition for finite spectrum assignment is obtained. Notice that the resulting feedback law involves integrals over the past control. In case of delays in state variables, a technique based on the finite Laplace transform leads to a constructive design procedure. The resulting feedback consists of proportional and (finite interval) integral terms over present and past values of state variables. In [27], a similar finite pole placement for time-delay systems with commensurate delays is proposed. Feedback laws defined in terms of Volterra equations are obtained owing to the properties of the Bezout ring of operators including derivatives, localized and distributed delays. Recently, in [2] it is shown that under appropriate conditions the assignment of exactly $P S_{B}$ negative distinct poles guarantees the exponential stability of the solution. The dominancy of the assigned poles is shown using a factorization technique. Other analytical/numerical placement methods for retarded time-delay systems are proposed in [33,36,47], see also [62] for further insights on pole-placement methods for retarded time-delay systems with proportional-integral-derivative controller-design.

In recent works, the characterization of multiple spectral values for time-delay systems of retarded type were established using a Birkhoff/Vandermonde-based approach; see for instance [7-9, 14]. In particular, in [8], it is shown that the admissible multiplicity of the zero spectral value is bounded by the generic Polya and Szegö bound denoted $P S_{B}$, which is merely the degree of the corresponding quasipolynomial ${ }^{1}$, see for instance [46]. In [9], it is shown that a given crossing imaginary root with a non vanishing frequency never reaches $P S_{B}$ and a sharper bound for its admissible multiplicities is established. Moreover, in [14], the manifold corresponding to a multiple root for scalar time-delay equations defines a stable manifold for the steady state. An example of a scalar retarded equation with two delays is studied in [9] where it is shown that the multiplicity of real spectral values may reach the $P S_{B}$. In addition, the corresponding system has some further interesting properties: (i) it is asymptotically stable, (ii) its spectral abscissa (rightmost root) corresponds to this maximal admissible multiple root located on the real axis. Such observations enhance the outlook of further exhibiting the

\footnotetext{
${ }^{1}$ The quasipolynomial degree is defined as the sum of degrees of the involved polynomials plus the corresponding number of delays
} 
existing links between the maximal admissible multiplicity of some negative spectral value reaching the quasipolynomial degree and the stability of the trivial solution of the corresponding dynamical system. This dominancy property induced by multiplicity appears also in optimization problems since such a multiple spectral value is indeed the rightmost root, see also [56].

In order to better understand the problem discussed in the paper as well as the solution we are proposing, consider the simple example of a scalar system controlled by a delayed output feedback which in the frequency domain reads as,

$$
\left\{\begin{aligned}
H_{y u}(s) & =\frac{1}{s-a} \\
C_{\tau}(s) & =k e^{-\tau s}
\end{aligned}\right.
$$

where $H_{y u}$ and $C_{\tau}$ are respectively the open-loop plant and the controller. It is easy to see that the stability of the closed-loop system is subordinate to the location of the characteristic roots of the following quasipolynomial function:

$$
Q(s, \tau)=s-a+k e^{-s \tau}
$$

which involves three parameters $(a, k, \tau) \in \mathbb{R}^{2} \times \mathbb{R}_{+}$. The vanishing of the delay $\tau$ allows to assign the unique closedloop root using the gain $k$. However, if $\tau$ is non zero then the closed-loop admits an infinite number of roots, for which the gain $k$ can be set to assign the corresponding dominant characteristic root as proposed in [22] using the Lambert $\mathcal{W}$ function properties, see also [61] for further insights on this special function. The extension of the rightmost root assignment problem to second order plants is not straightforward, which is essentially due to the increase of both the number of parameters ( 5 parameters) and the degree of the corresponding quasipolynomial.

The paper is organized as follows. Section 2 presents some prerequisites in complex analysis and states some technical Lemmas on the behavior of parametric trigonometric polynomials encountered in the proof of the main results. Section 3 provides some motivating examples emphasizing the interest of better understanding the effect of multiple spectral values on the stability of delay systems. The main results are presented in Section 4 where a discriminant-based parametric analysis of MID is provided. Section 5 focuses on the sensitivity of the proposed design in presence of uncertainties. Section 6 exhibits some applicative perspectives of the dominant-root assignment we propose. The problems of stabilizing a double integrator via a delayed PD-controller and the stabilizing an inverted pendulum on cart as well as the regulation of the Mach number in a wind tunnel are numerically investigated and discussed. Finally, Section 7 opens new leads for future developments. Indeed, the investigation of MID for time-delay system of neutral type is still an open problem.

\section{PROBLEM STATEMENT AND PREREQUISITES}

Consider the generic time-delay system of retarded type with a single time delay:

$$
\dot{\xi}=A_{0} \xi(t)+A_{1} \xi(t-\tau)
$$

where $\xi=\left(\xi_{1}, \ldots, \xi_{n}\right) \in \mathbb{R}^{n}$ is the state-vector, under appropriate initial conditions, belonging to the Banach space of continuous functions $\mathcal{C}\left(\left[-\tau_{N}, 0\right], \mathbb{R}^{n}\right)$. Here, $\tau$ is a positive constant delay and $A_{j} \in \mathcal{M}_{n}(\mathbb{R})$ for $j=0 \ldots 1$ are real valued matrices.

It is well known that the asymptotic behavior of the solutions of (2) is determined from the spectrum $\aleph$ designating the set of eigenvalues of the characteristic matrix function : $\mathbb{C} \times \mathbb{R}_{+}^{*}$ defined by:

$$
\mathbb{M}(s, \tau)=s I-A_{0}-A_{1} e^{-\tau s}
$$

Furthermore, these eigenvalues are the roots of the associated characteristic function which is a quasipolynomial $Q$ : $\mathbb{C} \times \mathbb{R}_{+} \rightarrow \mathbb{C}$ of the form:

$$
Q(s, \tau)=\operatorname{det} \mathbb{M}(s, \tau)
$$


In this paper, we restrict our analysis to $n=2$ and so the considered class of quasipolynomial functions is given by:

$$
Q(s, \tau)=P_{0}(s)+P_{1}(s) e^{-\tau s},
$$

with

$$
\left\{\begin{array}{l}
P_{0}(s)=s^{2}+a_{1} s+a_{0} \\
P_{1}(s)=\alpha_{1} s+\alpha_{0}
\end{array}\right.
$$

Some generic result on the location of associated spectral values for arbitrary $n$ can be found in [5]; see also [34], where, in particular, the proof of the proposition below is given.

Proposition 1. If $s$ is a characteristic root of system (2), then it satisfies

$$
|s| \leq\left\|A_{0}+A_{1} e^{-\tau s}\right\|_{2} .
$$

The above property combined with the triangular inequality provides a generic envelope curve around the characteristic roots corresponding to system (2), see for instance [38] for further insights on spectral envelopes for retarded time-delay systems with a single delay.

The goal of this work is twofold. First, it characterizes the admissible/maximal spectral values' multiplicities and it describes their effect on the stability of the trivial solution as well as the corresponding exponential decay rate, see for instance [37]. Second, it emphasizes links between the location of the poles of the open-loop system and the rightmost roots of the closed-loop system. It is worth mentioning that in the case of linear second-order systems of retarded type, the corresponding 5 parameters generate complexity in the analysis. As a byproduct of the approach, the main steps of an algorithmic procedure are proposed. We think that such an idea can be extended to more general quasipolynomials.

\subsection{On integration contours for quasipolynomials corresponding to retarded systems}

Cauchy's argument principle is a basic complex analysis property widely used in the stability analysis of linear timeinvariant dynamical systems, see for instance [31,50]. Roughly speaking, the argument principle establishes a correspondance between the number of zeros minus the number of poles of a meromorphic function $f$ in a simply connected domain $D \subset \mathbb{C}$ and a contour integral, on the boundary $\partial D$, of the function's logarithmic derivative, which is also the winding number of the curve $\partial D$. Several stability methods such as the Nyquist criterion and the Mikhaylov curve derive from the argument principle, see for instance $[35,41]$ and $[44,45]$ for more contemporary applications of these methods.

Theorem 2.1 ([1]). Let $U$ be a simply connected region with boundary $\Gamma$ (piecewise smooth and oriented anti-colockwise). Let $f$ be a meromorphic function in an open set containing the closure $\bar{U}$ with poles $p_{1}, \ldots, p_{l}$ and zeros $s_{1}, \ldots, s_{m}$ counted according to their multiplicity, none of which belonging to the closed curve $\Gamma$. Then

$$
\frac{1}{2 i \pi} \oint_{\Gamma} \frac{f^{\prime}(s)}{f(s)} d s=\mathcal{Z}-\mathcal{P},
$$

where $\mathcal{Z}$ and $\mathcal{P}$ designate respectively the number of zeros and the number of poles of $f$ enclosed by $\Gamma$.

In particular, note that if $f$ is a holomorphic function (in our case a quasipolynomial function) and $\Gamma$ is a closed piecewise $C^{1}$ curve then

$$
\frac{1}{2 i \pi} \oint_{\Gamma} \frac{f^{\prime}(s)}{f(s)} d s=\mathcal{Z} .
$$

Moreover, standard contours like the modified Bromwich contour are often used to explore the asymptotic stability of dynamical systems' solutions, as it allows to count the zeros in $\Re(s)>0$; see for instance Figure 1. Other contours can be chosen based on the inherent properties of the considered characteristic function; see for instance Figure 2. Practically, owing to Proposition 1, a generic supremum bound for the real and imaginary parts of the roots of function (4) is established. Then, one may define an integration contour $\Gamma=\cup_{k=1}^{l} g_{k}$ which is not passing through zeros of $Q$ and taken as a counterclockwise closed curve. Hence, the contour integral over $\Gamma$ is defined as the sum of the integrals over the directed smooth curves that make up $\Gamma$, as illustrated in Figures 2 for instance. 

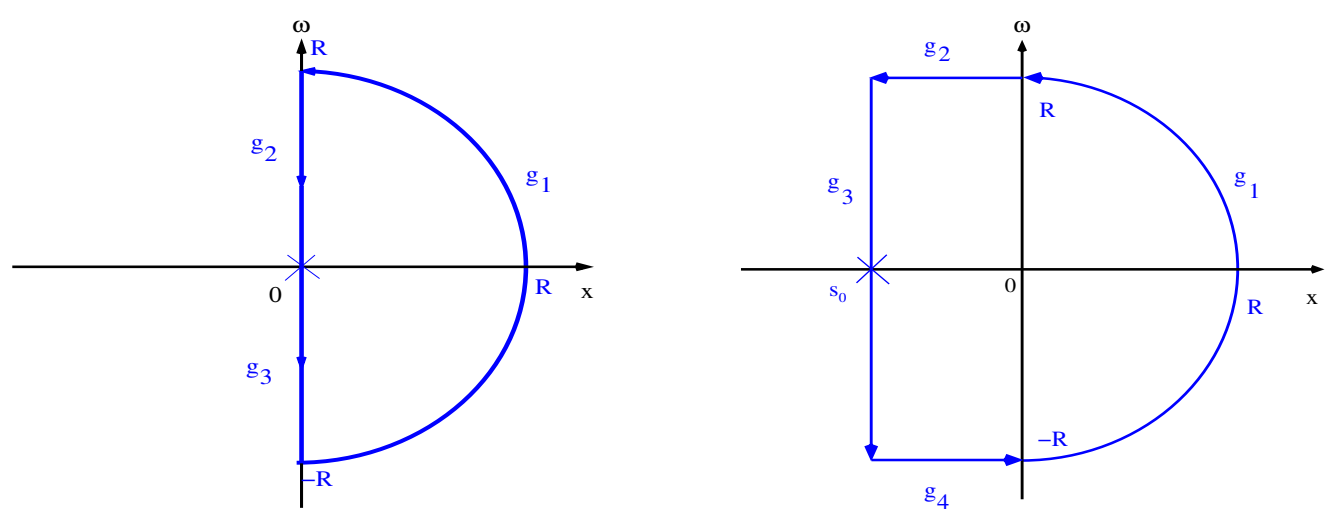

FIGURE 1. Generic contours for applying the argument principle to investigate qualitative properties of a linear time-invariant dynamical system. The argument principle allows to count the difference between the number of zeros and the number of poles of a given meromorphic function inside the blue closed contour. (Left) The standard modified Bromwich contour usually used for asymptotic stability investigation. (Right) A contour often used to investigate $\alpha$-stability or the dominancy of a given root.

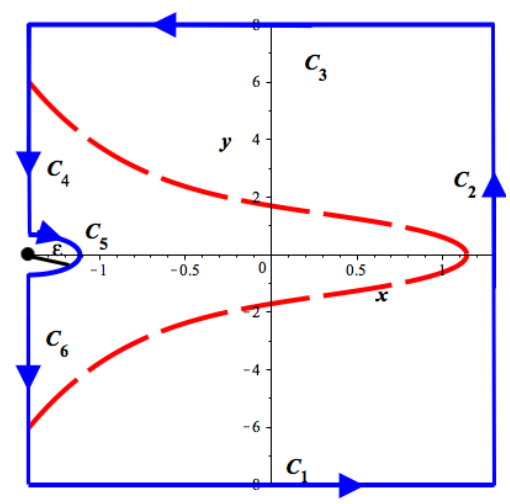

FIGURE 2. The simplified contour used in [10] for applying the argument principle in order to investigate the dominancy of the multiple root is the solid blue curve. The generic spectrum envelope established in Proposition 1 is the dashed red curve for equation (12) under conditions (11) where $a_{0}=\tau=1$.

\subsection{Some properties of quasipolynomials}

In order to apply the argument principle and count the number of zeros of a quasipolynomial in $\Re(s)>0$, one begins by exploring the corresponding imaginary roots as well as their respective multiplicities. Efficient methods for crossing imaginary roots identification exist; for instance, one may use the Rekasius substitution $[43,48]$ or matrix pencil [34] to mention only a few, see also [9] for investigating the multiplicity of such roots. Also, note that real and imaginary parts of quasipolynomials consist in trigonometric polynomials having a parity property. In particular, in second-order delay systems (4), one has

$$
\left\{\begin{array}{l}
\Re(Q(i \omega, \tau))=-\omega^{2}+a_{0}+\alpha_{0} \cos (\omega \tau)+\alpha_{1} \omega \sin (\omega \tau), \\
\Im(Q(i \omega, \tau))=a_{1} \omega+\alpha_{1} \omega \cos (\omega \tau)-\alpha_{0} \sin (\omega \tau),
\end{array}\right.
$$

where the real and the imaginary parts are respectively even and odd with respect to $\omega$, which is a useful property for counting the quasipolynomial's roots in the right-half plane; see [21]. 


\subsection{Technical lemmas}

Hereafter, we state some lemmas pertaining to some properties of a particular class of parametric-trigonometric polynomials encountered in the proof of the main results. The proofs of the lemmas are presented in the Appendix.

Lemma 1. Consider the transcendental function defined by:

$$
F(\rho)=6 \cos (\rho)+2 \rho \sin (\rho)+\rho^{2}-6
$$

admits a unique real solution $\rho^{\star}$ which is located in the interval $] \pi, \frac{3 \pi}{2}[$.

Lemma 2. Let $\zeta$ be a real non-negative parameter and consider the transcendental functions defined by :

$$
\left\{\begin{array}{l}
F_{+}(\rho)=\rho^{2}+(6-2 \zeta)(\cos (\rho)-1)+(2-\zeta) \rho \sin (\rho), \\
G_{+}(\rho)=(2-\zeta) \rho \cos (\rho)+(2 \zeta-6) \sin (\rho)+(4-\zeta) \rho .
\end{array}\right.
$$

A positive real number $\rho^{*}$ is a root of $F_{+}$if, and only if, there exists $\zeta^{*}>0$ such that

$$
\zeta^{*}=\frac{F\left(\rho^{*}\right)}{2\left(\cos \left(\rho^{*}\right)-1\right)+\rho^{*} \sin \left(\rho^{*}\right)}
$$

where $F$ is the transcendental function studied in Lemma 1. Furthermore, the following assertions hold:

i) To every $\left.\zeta^{*} \in\right] 0,1\left[\right.$ corresponds a unique $\rho^{*}>0$ root of $F_{+}$and one has $G_{+}\left(\rho^{*}\right)>0$.

ii) To every $\zeta^{*}>1$ corresponds an even number $n_{\zeta^{*}}$ (including zero and counting multiplicity) of roots of $F_{+}$for which $G_{+}$is negative.

Lemma 3. Let $\zeta$ be a real non-negative parameter and consider the transcendental functions defined by:

$$
\left\{\begin{array}{l}
F_{-}(\rho)=\rho^{2}+(2 \zeta+6)(\cos (\rho)-1)+(2+\zeta) \rho \sin (\rho), \\
G_{-}(\rho)=(2+\zeta) \rho \cos (\rho)-(2 \zeta+6) \sin (\rho)+(4+\zeta) \rho .
\end{array}\right.
$$

To every positive number $\zeta^{*}$ corresponds an odd number of positive roots of $F_{-}$. Furthermore, the first root belongs to the interval $] 0,2 \pi[$.

\section{COMPREHENSIVE MOTIVATING EXAMPLES}

In this section, we focus on three case studies. The first one corresponds to the simplest delay differential model by which one can exhibit and explain in a comprehensive way the stability induced when forcing some particular spectral value to be multiple. The second, which in appearance illustrates the limitation of such a property, gives deep insights on conditions for such a property to apply.

\subsection{Exponential decay rate for a scalar equation with a single delay}

The starting point of this work in progress and the first analytical proof of the multiplicity-induced dominancy was proposed in [14]. Indeed, a simple scalar differential equation with one delay representing a biological model describing the dynamics of a vector-borne disease was considered. In its linearized version, the infected host population $\xi(t)$ is governed by:

$$
\dot{\xi}(t)+a_{0} \xi(t)+a_{1} \xi(t-\tau)=0
$$

where $a_{1}>0$ designates the contact rate between infected and uninfected populations assuming that the infection of the host recovery proceeds exponentially at a rate $-a_{0}>0$, see for instance [16] and [49] for other time-delay models issued 
from system biology. It was shown in [14] that for a given positive delay, equation (10) admits a double spectral value at $s=s_{0}$ if, and only if,

$$
s_{0}=-\frac{a_{0} \tau+1}{\tau} \text { and } a_{1}=\frac{\mathrm{e}^{s_{0} \tau}}{\tau} .
$$

In addition, it was emphasized that $s_{0}$ is the rightmost root, and that if $s_{0}<0$ then the zero solution of system (10) is asymptotically stable; see [15] for general results on the stability of a scalar equation with a single delay.

Now, let us recall that $s=s_{0}$ is a spectral value of (10) if, and only if, $s_{0}$ is a root of the characteristic equation

$$
Q(s, \tau)=s+a_{0}+a_{1} e^{-s \tau} .
$$

The main ingredient of the dominancy proof of $s_{0}$ is an integral equation which cannot be satisfied for any spectral value $s$ with $\Re(s)>s_{0}$. Namely, it was shown that if $a_{1}$ satisfies (11), then the characteristic function reads:

$$
Q(s, \tau)=\left(s-s_{0}\right)\left(1-\int_{0}^{1} e^{-\tau\left(s-s_{0}\right) t} d t\right) .
$$

As a matter of fact, if $s_{1}=\zeta+j \eta \neq s_{0}$ is a root of (13) then $s_{1}$ is a root of its second factor. Hence, we obtain

$$
1=\int_{0}^{1} e^{-\tau\left(\zeta-s_{0}\right) t} d t
$$

But, $e^{-\tau\left(\zeta-s_{0}\right) t}<1$ for $\zeta-s_{0}>0$ and $0<t<1$, thereby exhibiting the dominancy of $s_{0}$.
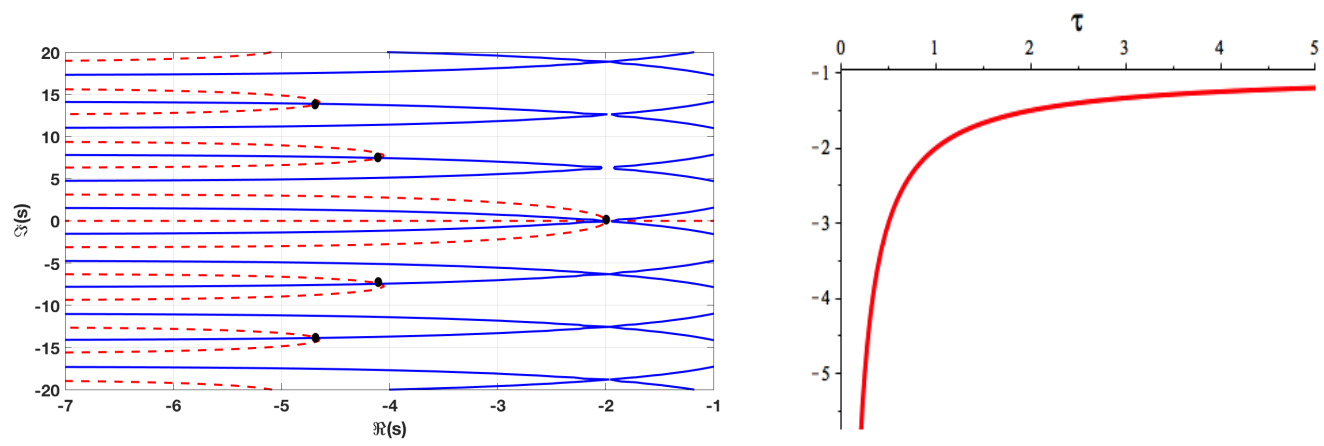

FIGURE 3. (Left panel) The distribution of the spectrum corresponding to equation (12) and system (11) for $a_{0}=\tau=1$ and $a_{1}=e^{-2}$. The roots' distribution is illustrated using QPmR toolbox from [57]. (Right panel) The rightmost root corresponding to equation (12) and system (11) as a function of the delay $\tau$ for $a_{0}=1$ and $a_{1}=\frac{\mathrm{e}^{-(\tau+1)}}{\tau}$.

Remark 1. The rightmost root $s_{0}$ corresponding to equation (12), where system (11) is satisfied, varies in the interval $\left.s_{0} \in\right]-\infty,-a_{0}[$. Figure 3 illustrates the behavior of the rightmost root with respect to the time-delay variation.

\subsection{Multiple spectral values for time-delay systems are not necessarily dominant}

The problem of stabilization of a chain of integrators is considered in [39] where a single integrator can be stabilized by a single delay state-feedback. Indeed, a positive gain guarantees the closed-loop stability of the system free of delay, and, by continuity, there exists a (sufficiently small) delay in the output preserving the stability of the closed-loop system. However, the situation is completely different for a chain of integrators of order $n$ when $n>1$. For instance, consider the time-delay system characterized by the following quasipolynomial function:

$$
Q(s, \tau)=s^{2}+\alpha e^{-\tau s} \text {. }
$$




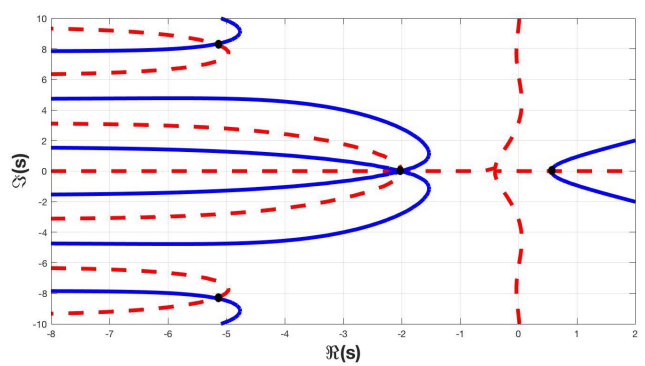

FIGURE 4. Sparsity-induced loss of dominancy for the multiple spectral value. Each intersection between the solid blue/dashed red curves corresponds to a spectral value of function (17). For $\tau=1$, the dominancy of $s_{1} \approx 0.557$ with respect to the double root at $s_{0}=-2$ is illustrated. The roots' distribution is illustrated using QPmR toolbox from [57].

It can be checked that the maximal admissible multiplicity is 2 and it can be attained if, and only if,

$$
\alpha=-4 \frac{\mathrm{e}^{-2}}{\tau^{2}}, s=-\frac{2}{\tau} .
$$

However, the main result from [39] asserts that either $n$ distinct delays or a proportional+delay compensator with $n-1$ distinct delays are sufficient to stabilize a chain including $n$ integrators. In [24], a like assertion is shown to be also necessary to stabilize the chain of $n$ integrators. Hence, in our case, either 2 distinct delays or a proportional+delay are necessary and sufficient to stabilize the double integrator. In conclusion, there exists at least a spectral value for (15) with a positive real part. As a result, $s_{0}=-\frac{2}{\tau}$, while being a multiple root, it is not dominant. Indeed, consider (15)-(16) with $\tau=1$, that is

$$
Q(s, 1)=s^{2}-4 e^{-(s+2)} .
$$

As illustrated in Figure 4, the dominancy property is lost since $s_{1} \approx 0.557$ is a root of function (17). This is justified by the sparsity of (17).

\subsection{Stabilizing an oscillator via a delayed output-feedback}

Consider the stability of the trivial solution corresponding to the control system

$$
\left\{\begin{array}{l}
\ddot{\xi}(t)+a_{1} \dot{\xi}(t)+a_{0} \xi(t)=u(t), \\
\phi(t)=\gamma \xi(t)
\end{array}\right.
$$

where $\phi(t)$ is the system output and $u(t)=\frac{\beta}{\gamma} \xi(t-\tau)$ is the control law, which amounts to studying the roots of the quasipolynomial function

$$
Q(s, \tau)=s^{2}+a_{1} s+a_{0}+\beta e^{-\tau s} .
$$

Using the standard linear change of variables $s=\frac{a_{1} \lambda}{2}$, one obtains the normalized characteristic function

$$
\left\{\begin{aligned}
\tilde{Q}(\lambda, \tilde{\tau}) & =\lambda^{2}+2 \lambda+a_{0}+\alpha e^{-\lambda \tilde{\tau}}, \text { where } \\
\alpha & =\frac{4}{c_{1}^{2}} \beta_{0}, \tilde{\tau}=\frac{c_{1}}{2} \tau \text { and } a_{0}=4 \frac{c_{0}}{c_{1}{ }^{2}} .
\end{aligned}\right.
$$

If $\alpha=0$, the spectral abscissa is minimized at $a_{0}=1$ which corresponds to to the rightmost root located at $\lambda_{0}=-1$, see for instance [25]. By exploiting the delay effect, the following proposition proved in [13] asserts that the solution's decay rate can be further improved by decreasing the corresponding rightmost root. Assume that $a_{0}>1$, then the following proposition holds. 
Theorem 3.1 ([13]).

i) The multiplicity of any given root of the quasipolynomial function (19) is bounded by 3 .

ii) The quasipolynomial (19) admits a real spectral value at $z_{0}=-1-\frac{1}{\tilde{\tau}}$ with algebraic multiplicity 3 if, and only if,

$$
\tilde{\tau}=\sqrt{\frac{1}{a_{0}-1}} \text { and } \alpha=-\frac{2 e^{-(1+\tilde{\tau})}}{\tilde{\tau}^{2}} .
$$

iii) If equations (20) are satisfied then $z=z_{0}$ is the rightmost root of function (19).

Remark 2. If equalities (20) are satisfied then the trivial solution of the second-order equation $\ddot{\xi}(t)+2 \dot{\xi}(t)+a_{0} \xi(t)=$ $\alpha \xi(t-\tilde{\tau})$ is asymptotically stable with $\xi(t) \approx e^{z_{0} t}$.

\section{MAin Result: PARAMETRiC MID FOR SECOND-ORDER SYSTEMS}

Second-order linear systems capture the dynamic behavior of many natural phenomena and have found numerous applications in a variety of fields, such as vibration and structural analysis. Stabilization of solutions to such a reduced order model represents a standard test bench to approve of new paradigms and methodologies in control design.

The problem we consider is the characterization of the admissible multiplicities of spectral values and their effect on stability of the time-delay system $\ddot{\xi}(t)+a_{1} \dot{\xi}(t)+a_{0} \xi(t)+\alpha_{0} \xi(t-\tau)+\alpha_{1} \dot{\xi}(t-\tau)=0$. It is worth mentioning that such a system depicts several unexpected properties and in particular, for each choice of the coefficients, the delay parameter induces a stabilizing/destabilizing behavior. In other words, increasing the delay value may be beneficial for the system's dynamics. Such a property does not hold for scalar systems with one delay, for which increasing the delay leads to instability, see for instance [40,58].

From a control theory viewpoint, the aim is to establish a delayed-state-feedback controller $u(t)=-\alpha_{0} \xi(t-\tau)-$ $\alpha_{1} \dot{\xi}(t-\tau)$ able to stabilize solutions of the following control system:

$$
\ddot{\xi}(t)+a_{1} \dot{\xi}(t)+a_{0} \xi(t)=u(t) .
$$

In the Laplace domain, the latter generic control problem yields the following characteristic quasipolynomial function.

$$
Q(s, \tau)=P_{0}(s)+P_{1}(s) e^{-\tau s},
$$

where $P_{0}(s)=s^{2}+a_{1} s+a_{0}$ and $P_{1}(s)=\alpha_{1} s+\alpha_{0}$ as defined in (6).

From an algebraic geometry viewpoint, it is consistent to parametrize such a control problem via the discriminant of the characteristic polynomial related to the open-loop (uncontrolled) equation. Namely, the complex/real nature of the roots of the polynomial $P_{0}(s)$ has a strong effect on the characterization of the controller's gains and delay enabling a desired fast stabilization using the MID property. In the sequel, it shall be emphasized that $\Delta=a_{1}^{2}-4 a_{0}$, the discriminant of $P_{0}$, defines an efficient and necessary criterion to exhibit the potential applicability and the limitations of the proposed methodology. Indeed, a discriminant-based parametric delayed-output-feedback controller design is established. Our analysis splits following the sign of the said discriminant.

\subsection{Open-loop system with one oscillating mode}

One oscillating mode as solution of the uncontrolled equation (two conjugate complex roots) corresponds, from a purely algebraic viewpoint, to a characteristic polynomial with a strictly negative discriminant $\Delta$. In such a case, the following theorem gives a bound for quasipolynomial root's multiplicity and provides the explicit MID-based controller's gains and delay.

Theorem 4.1. Considering equation (4), the following assertions hold.

i) The multiplicity of any given root of the quasipolynomial function (4) is bounded by 4, it can only be achieved on the real axis and under negativity of $\Delta$. 


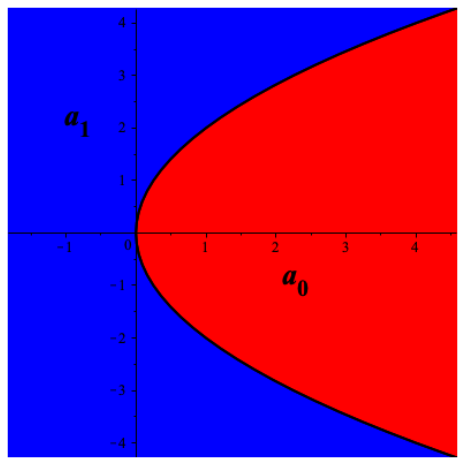

FIGURE 5. Two complementary regions in the parametric space separated by the parabola $a_{1}^{2}-4 a_{0}=0$. Each point of the parabola corresponds to a double root. To each point of the blue region corresponds a real-rooted quadratic polynomial. In the red region the corresponding $P_{0}$ admits two conjugate complex roots.

ii) The quasipolynomial (4) admits a real spectral value at $s=s_{0}$ with algebraic multiplicity 4 if, and only if,

$$
s_{0}=-\frac{a_{1}+\sqrt{-2 \Delta}}{2}
$$

and the system parameters satisfy

$$
\tau=2 \sqrt{-\frac{2}{\Delta}}, \alpha_{0}=\frac{\left(5 \Delta-a_{1} \sqrt{-2 \Delta}\right)}{4} \mathrm{e}^{s_{0} \tau}, \alpha_{1}=-\frac{\sqrt{-2 \Delta}}{2} \mathrm{e}^{s_{0} \tau} .
$$

iii) If (23) is satisfied, then $s=s_{0}$ is the spectral abscissa corresponding to (4).

iv) If (23) is satisfied then the trivial solution of the closed-loop equation (21) is asymptotically stable if, and only if, either $\left(a_{1} \geq 0\right.$ and $\left.a_{0}>\frac{a_{1}^{2}}{4}\right)$ or $\left(a_{1}<0\right.$ and $\left.a_{0}>\frac{3 a_{1}^{2}}{8}\right)$.

Proof.

i) and ii). Note that the degree of the quasipolynomial function is equal to 4 as seen in previous sections.

First, the vanishing of the quasipolynomial $Q(s, \tau)$ yields the elimination of the exponential term as a rational function in $s$. As a matter of fact, setting $Q(s, \tau)=0$ yields

$$
\mathrm{e}^{-s \tau}=\frac{-s^{2}-a_{1} s-a_{0}}{\alpha_{0}+\alpha_{1} s}
$$

The substitution of the last result in the ideal generated by the first three derivatives $<\partial_{s} Q(s, \tau), \partial_{s}^{2} Q(s, \tau), \partial_{s}^{3} Q(s, \tau)>$ gives a system of algebraic equations in the variable $s$ and parametrized by $p=\left(\tau, a_{0}, a_{1}, \alpha_{0}, \alpha_{1}\right)$ :

$$
\left\{\begin{array}{l}
\alpha_{1} \tau s^{3}+\left(\alpha_{1}+\tau a_{1} \alpha_{1}+\tau \alpha_{0}\right) s^{2}+\left(\tau a_{0} \alpha_{1}+2 \alpha_{0}+\tau a_{1} \alpha_{0}\right) s \\
+\tau \alpha_{0} a_{0}+a_{1} \alpha_{0}-\alpha_{1} a_{0}=0 \\
\alpha_{1} \tau^{2} s^{3}+\left(\tau^{2} a_{1} \alpha_{1}-2 \tau \alpha_{1}+\tau^{2} \alpha_{0}\right) s^{2}+\left(\tau^{2} a_{0} \alpha_{1}-2 \tau a_{1} \alpha_{1}-2 \alpha_{1}+\tau^{2} a_{1} \alpha_{0}\right) s \\
-2 \alpha_{0}+\tau^{2} \alpha_{0} a_{0}-2 \tau a_{0} \alpha_{1}=0, \\
\alpha_{1} \tau^{3} s^{3}+\left(\tau^{3} a_{1} \alpha_{1}+\tau^{2}\left(\tau \alpha_{0}-3 \alpha_{1}\right)\right) s^{2}+\left(\tau^{3} a_{0} \alpha_{1}+\tau^{2} a_{1}\left(\tau \alpha_{0}-3 \alpha_{1}\right)\right) s \\
+\tau^{2} a_{0}\left(\tau \alpha_{0}-3 \alpha_{1}\right)=0 .
\end{array}\right.
$$


Using elimination, we deduce equality (22) and the values of the delay $\tau$ and the gain $\alpha_{0}$ both parametrized in $\alpha_{1}$ and the open-loop parameters $a_{0}$ and $a_{1}$ as follows

$$
\tau=2 \sqrt{-\frac{2}{\Delta}}, \alpha_{0}=\alpha_{1}\left(\frac{a_{1}}{2}-\frac{5}{4} \sqrt{-2 \Delta}\right) .
$$

Hence, substituting the last equalities in (24), we get the value of the gain $\alpha_{1}$ given in (23). Obviously, (23) applies only when $\Delta<0$. Finally, under (23) one easily checks that $\partial_{s}^{4} Q\left(s_{0}, \tau\right)=-16 / \Delta \neq 0$, which entails that the maximal multiplicity is 4 and cannot be attained at any $s \neq s_{0}$.

iii). Parametrization and scaling: Under (23), Equation (4) parametrized by the discriminant $\Delta$ reads as

$$
Q(s, \tau)=s^{2}+a_{1} s+a_{0}+\left(\frac{5 \Delta}{4}-\frac{a_{1} \sqrt{-2 \Delta}}{4}-\frac{s \sqrt{-2 \Delta}}{2}\right) \mathrm{e}^{-\frac{-2 s \sqrt{-2 \Delta-a_{1} \sqrt{-2 \Delta}+2 \Delta}}{\Delta}} .
$$

Using the change of variables $z=s-s_{0}$, the above expression reduces to

$$
\tilde{Q}(z, \tau)=z^{2}-\frac{4 z}{\tau}+\frac{6}{\tau^{2}}-\left(\frac{6}{\tau^{2}}+\frac{2 z}{\tau}\right) \mathrm{e}^{-z \tau} .
$$

Accordingly, the zeros of (26) are exactly the scaled zeros of (25) so that $s_{k}-s_{0}=z_{k}$, where $s_{0}$ is given in (22). Also, recall that $s_{0}$ is a root of (25) with multiplicity 4 which is equivalent to 0 being a root of (26) with multiplicity four. Finally, proving the dominancy of $s_{0}$ as a root of (25) is equivalent to proving the dominancy of 0 as a root of (26).

Characterizing imaginary roots of the scaled equation: In order to apply the argument principle on the standard modified Bromwich contour, allowing to count the roots of the quasipolynomial (26) on the right half-plane, a deflation which eliminates the roots on the imaginary axis is required. To do so, we first investigate nonzero imaginary roots of (26). Assuming that there exists $\omega>0$ such that $z=i \omega$ is a root of (26), we define $\mathcal{R}(\omega)=\Re\left(i^{-2} Q(i \omega)\right)$ and $\mathcal{S}(\omega)=$ $\Im\left(i^{-2} Q(i \omega)\right)$, so that

$$
\left\{\begin{array}{l}
\mathcal{R}(\omega)=6 \frac{\cos (\omega \tau)}{\tau^{2}}+2 \frac{\omega \sin (\omega \tau)}{\tau}+\omega^{2}-\frac{6}{\tau^{2}}, \\
S(\omega)=2 \frac{\omega \cos (\omega \tau)}{\tau}-6 \frac{\sin (\omega \tau)}{\tau^{2}}+4 \frac{\omega}{\tau} .
\end{array}\right.
$$

In consequence, for any $z=i \omega$, root of (26), we have $\mathcal{R}(\omega)=\mathcal{S}(\omega)=0$. Then, some algebraic manipulations allow to eliminate the trigonometric functions as follows

$$
\left\{\begin{array}{l}
\cos (\omega \tau)=-\frac{1}{2} \frac{7 \omega^{2} \tau^{2}-18}{\omega^{2} \tau^{2}+9} \\
\sin (\omega \tau)=-\frac{1}{2} \frac{\omega \tau\left(\omega^{2} \tau^{2}-18\right)}{\omega^{2} \tau^{2}+9}
\end{array}\right.
$$

Using the standard trigonometric identity $\cos ^{2}(\omega \tau)+\sin ^{2}(\omega \tau)=1$, one obtains exclusively 0 and the non-vanishing solutions $\omega= \pm \frac{3 i}{\tau}$, which are discarded since we are dealing with positive frequencies. Under Stepan-Hassard notations (see Appendix), we have $K=\kappa=4$ where $K$ designates the number of imaginary roots of (26) and $\kappa$ is the multiplicity of 0 . Hence, the deflated function which is integrated on the Bromwich contour $\mathcal{B}$ is given by

$$
\hat{Q}(z, \tau)=\frac{\tilde{Q}(z, \tau)}{z^{4}}
$$

It has the same zeros encircled by $\mathcal{B}$ as the quasipolynomial $\tilde{Q}$. Also, recall that the number of such zeros is nothing but the number of roots of $Q$ in the right-half plane $\left\{s \in \mathbb{C}\right.$, s.t. $\left.\Re(s)>s_{0}\right\}$. 
Characterizing $\omega_{k}$ the positive roots of $R$ and exploring the $\operatorname{sign}\left(\mathcal{S}\left(\omega_{k}\right)\right)$ : Let us explore the positive roots of $\mathcal{R}$. If $\omega>0$ is a root of the first transcendental function of (27), then

$$
6 \frac{\cos (\omega \tau)}{\tau^{2}}+2 \frac{\omega \sin (\omega \tau)}{\tau}+\omega^{2}-\frac{6}{\tau^{2}}=0 .
$$

Multiplying the last equation by $\tau^{2}$ reduces the problem to the search of zeros of an univariate function:

$$
F(\rho)=6 \cos (\rho)+2 \rho \sin (\rho)+\rho^{2}-6 \quad(\rho=\omega \tau)
$$

Now, Lemma 1 in Section 2, states that function $F$ admits a unique solution $\left.\rho^{\star} \in\right] \pi, \frac{3 \pi}{2}[$ which is equivalent to state that for a given positive delay $\tau^{\star}$ there exists a unique frequency $\omega^{\star} \in \mathbb{R}_{+}^{*}$ such that $\rho^{\star}=\omega^{\star} \tau^{\star}$ and $\mathcal{R}\left(\omega^{\star}\right)=0$.

Let us define $G(\rho)=\tau^{2} \mathcal{S}(\omega)$ in the same fashion. Then,

$$
G(\rho)=2 \rho(2+\cos (\rho))-6 \sin (\rho)
$$

which is positive in the interval $] \pi, 2 \pi\left[\operatorname{since} \sin (\rho)<0\right.$. In particular, one has $G\left(\rho^{\star}\right)>0$ which means that $\mathcal{S}\left(\omega^{\star}\right)>0$; see Figure 6 for illustration.

Counting the number of roots dominating $s_{0}$ : We are able now to compute the number of unstable roots for (26) based on the computation of the argument variation. As a matter of fact, combining all the above collected information on the behavior of both the real $\mathcal{R}$ and the imaginary $\mathcal{S}$ parts of the quasipolynomial function (26), we apply the Stepan-Hassard formula [21,54]; further details can be found in the Appendix. Namely, $\mathcal{Z}$ designates the number of roots dominating $s_{0}$ :

$$
\mathcal{Z}=\frac{n-K}{2}+\frac{1}{2}(-1)^{r} \operatorname{sign} \mathcal{S}^{(\kappa)}(0)+\sum_{j=1}^{r}(-1)^{j-1} \operatorname{sign} \mathcal{S}\left(\omega_{j}\right)
$$

where $n$ is the order of the system, $\kappa$ is the multiplicity of 0 as a root of (26), $K$ is the total number of roots of (26) on the imaginary axis and $r$ is the number of positive real roots of $\mathcal{R}$. In our case, $n=2, \kappa=K=4, r=1$ and $\mathcal{S}^{(4)}(\omega)=2 \sin (\omega \tau) \tau^{2}+2 \omega \cos (\omega \tau) \tau^{3}$ which vanishes at $\omega=0$. As a result, we easily obtain that $\mathcal{Z}=0$ owing to formula (29). Hence, the dominancy of 0 as a root of (26), equivalently the dominancy of $s_{0}$ as a root of (25), is proved.

iv). Condition $a_{0}>\frac{a_{1}^{2}}{4}$ guarantees the negativity of the discriminant $\Delta$. One easily sees that $a_{1}<0$ and $\left.a_{0} \in\right] \frac{a_{1}^{2}}{4}, \frac{3 a_{1}^{2}}{8}$ [ are inconsistent with $s_{0}<0$. Since assertion iii) states the dominancy of $s_{0}$, then, under (23), the spectrum corresponding to (4) consists of complex values with negative real parts only, which proves the asymptotic stability of the trivial solution.

\subsection{Open-loop system with non oscillating modes}

In this section we consider the case where the uncontrolled equation admits two real spectral values. The previous section showed that under such a configuration, the MID based-design cannot be applied by exploiting the maximal multiplicity which is equal to 4 , see Theorem 4.1. The following Theorem gives a bound for the quasipolynomial roots' multiplicity for $\Delta \geq 0$. In addition, it explicitly provides the MID-based controller's gains and delay by exploiting a lower multiplicity.

Theorem 4.2. Considering equation (4), the following assertions hold.

i) If the discriminant $\Delta \geq 0$, then the multiplicity of any given root of the quasipolynomial function (4) is bounded by 3.

ii) For an arbitrary positive delay $\tau$, the quasipolynomial (4) admits a real spectral value at $s=s_{ \pm}$with algebraic multiplicity 3 if, and only if,

$$
s_{ \pm}=\frac{-\tau a_{1}-4 \pm \sqrt{8+\tau^{2} \Delta}}{2 \tau},
$$



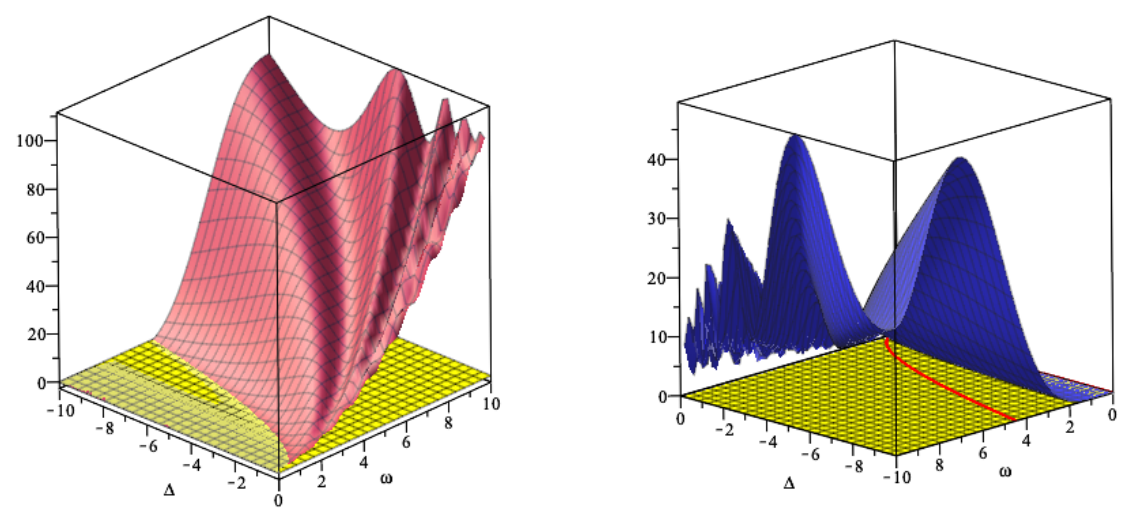

FIGURE 6. (Left) The existence of a unique solution of $\mathcal{R}$ given in (27) and parametrized by the negative left-free discriminant $\Delta$ and in red the 3D plot of $\mathcal{R}$ and in yellow the identically vanishing hyperplan. (Right) The positivity of $\mathcal{S}\left(\rho^{\star}\right)$ given (27) parametrized by the negative left-free discriminant $\Delta$. In blue the 3D plot of $\mathcal{S}$ and the red curve corresponds to the intersection of $\mathcal{R}$ with the identically vanishing hyperplan in yellow.

and the system parameters satisfy:

$$
\left\{\begin{array}{l}
\alpha_{0}=\left(a_{1} s_{ \pm}+\frac{a_{1}^{2}}{2}-\frac{\Delta}{2}+\frac{6 a_{1}+10 s_{ \pm}}{\tau}+\frac{6}{\tau^{2}}\right) \mathrm{e}^{s_{ \pm} \tau} \\
\alpha_{1}=\left(2 s_{0}+a_{1}+\frac{2}{\tau}\right) \mathrm{e}^{s_{ \pm} \tau}
\end{array}\right.
$$

iii) If $\left(\star_{+}\right)$(respectively $\left(\star_{-}\right)$) is satisfied then $s=s_{+}$is the spectral abscissa corresponding to (4) (respectively $s_{-}$ cannot be the spectral abscissa corresponding to (4)). Furthermore, for an arbitrary delay $\tau$ the multiple spectral value at $s_{-}$is always dominated by a single real root $s_{0}$.

iv) If $\left(\star_{+}\right)$is satisfied then the trivial solution is asymptotically stable if, and only if, $\tau$ satisfies the following conditions

$$
\left\{\begin{array}{l}
\tau \in] 0, \tau_{-}\left[\text {when } a_{0}<0,\right. \\
\text { or } \\
\tau \in] 0, \tau_{-}[\cup] \tau_{+}, \infty\left[\text { when } a_{0}>0 \text { and } a_{1}<0,\right.
\end{array}\right.
$$

where $\tau_{ \pm}=\frac{-a_{1} \pm \sqrt{\Delta+2 a_{0}}}{a_{0}}$.

Remark 3. The second and third assertions of Theorem 4.2 hold for negative discriminant $-\frac{8}{\tau^{2}}<\Delta<0$. Moreover, when $\Delta=-\frac{8}{\tau^{2}}$ the triple root at $s_{ \pm}$becomes the quadruple root prescribed in Theorem 4.1.

Proof.

i) and ii). As shown in assertion i) of Theorem 4.1, the degree of the quasipolynomial is equal to 4 which is an admissible multiplicity for any complex root of $Q(s, \tau)$ since $\Delta \geq 0$. So, we explore roots of multiplicity 3 of the quasipolynomial $Q(s, \tau)$. Again, we use (24) to eliminate the exponential term by writing it down as a rational function in $s$. Next, substituting (24) in the ideal generated by the first two derivatives $<\partial_{s} Q(s, \tau), \partial_{s}^{2} Q(s, \tau)>$ gives a system of algebraic 
equations in the variable $s$ and parametrized by $\left(\tau, a_{0}, a_{1}, \alpha_{0}, \alpha_{1}\right)$ as follows:

$$
\left\{\begin{array}{l}
\alpha_{1} \tau s^{3}+\left(\alpha_{1}+\tau a_{1} \alpha_{1}+\tau \alpha_{0}\right) s^{2}+\left(\tau a_{0} \alpha_{1}+2 \alpha_{0}+\tau a_{1} \alpha_{0}\right) s \\
+\tau \alpha_{0} a_{0}+a_{1} \alpha_{0}-\alpha_{1} a_{0}=0 \\
\alpha_{1} \tau^{2} s^{3}+\left(\tau^{2} a_{1} \alpha_{1}-2 \tau \alpha_{1}+\tau^{2} \alpha_{0}\right) s^{2}+\left(\tau^{2} a_{0} \alpha_{1}-2 \tau a_{1} \alpha_{1}-2 \alpha_{1}+\tau^{2} a_{1} \alpha_{0}\right) s \\
-2 \alpha_{0}+\tau^{2} \alpha_{0} a_{0}-2 \tau a_{0} \alpha_{1}=0 .
\end{array}\right.
$$

Solving the latter system and taking into account that $\tau>0$, we identify the couple $s_{ \pm}$of admissible triple roots listed in (30) parametrized in $\tau, a_{0}$ and $a_{1}$ and the value of the gain $\alpha_{0}$ parametrized in $\tau, \alpha_{1}, a_{0}, a_{1}$ where the latter can be written in the following compact form:

$$
\alpha_{0}=\frac{\alpha_{1}\left(\left(\tau^{2} a_{1}+10 \tau\right) s_{ \pm}+6+6 \tau a_{1}+2 \tau^{2} a_{0}\right)}{\tau\left(2 \tau s_{ \pm}+\tau a_{1}+2\right)}
$$

Finally, using (24) once more, we obtain the value of the gain $\alpha_{1}$ then $\alpha_{0}$ as stated in $\left(\star_{ \pm}\right)$.

Now, under $\left(\star_{ \pm}\right)$one easily checks that $\partial_{s}^{3} Q\left(s_{ \pm}, \tau\right)=\tau \sqrt{8+\tau^{2} \Delta} \neq 0$ entailing that the maximal multiplicity is 3 and that it is not be attained by any $s \neq s_{ \pm}$.

iii) New parametrization and scaling: Under $\left(\star_{ \pm}\right)$, Equation (4) parametrized by $\zeta=\sqrt{8+\tau^{2} \Delta}$ reads as:

$$
Q(s, \tau)=\left(\frac{\tau a_{1}-14 \pm 5 \zeta+s_{ \pm} \tau^{2} a_{1}+2 \tau^{2} a_{0}}{\tau^{2}}-\frac{(2 \mp \zeta) s}{\tau}\right) \mathrm{e}^{-\tau\left(s-s_{ \pm}\right)}+s^{2}+a_{1} s+a_{0},
$$

Using the change of variables $z=s-s_{ \pm}$allows to write the last quasipolynomial function as follows:

$$
\tilde{Q}(z, \tau)=z^{2}+\frac{( \pm \zeta-4) z}{\tau}+\frac{6 \mp 2 \zeta}{\tau^{2}}+\left(\frac{( \pm \zeta-2) z}{\tau}+\frac{ \pm 2 \zeta-6}{\tau^{2}}\right) \mathrm{e}^{-z \tau}
$$

Characterization of imaginary roots: In order to apply the argument principle on the Bromwich-like contour, to count the roots of the quasipolynomial (33) on the right half-plane, we first introduce a deflation that eliminates the roots on the imaginary axis, as priorly done in the proof item iii) of Theorem 4.1. To do so, we start by investigating nonzero imaginary roots for (33). Assuming that there exists $\omega>0$ such that $z=i \omega$ is a root of (33), we define $\mathcal{R}(\omega)=\Re\left(i^{-2} Q(i \omega)\right)$ and $\mathcal{S}(\omega)=\Im\left(i^{-2} Q(i \omega)\right)$ which yields :

$$
\left\{\begin{array}{l}
\mathcal{R}(\omega)=\frac{(6 \mp 2 \zeta) \cos (\omega \tau)}{\tau^{2}}+\frac{(2 \mp \zeta) \omega \sin (\omega \tau)}{\tau}+\omega^{2}-\frac{6 \mp 2 \zeta}{\tau^{2}}, \\
\mathcal{S}(\omega)=\frac{(2 \mp \zeta) \omega \cos (\omega \tau)}{\tau}-\frac{(6 \mp 2 \zeta) \sin (\omega \tau)}{\tau^{2}}+\frac{(4 \mp \zeta) \omega}{\tau} .
\end{array}\right.
$$

In consequence, for any $z=i \omega$, root of (33), we have $\mathcal{R}(\omega)=\mathcal{S}(\omega)=0$. Then, some algebraic manipulations allow to eliminate the trigonometric functions as follows:

$$
\left\{\begin{array}{l}
\cos (\omega \tau)=-\frac{\left(14 \mp 8 \zeta+\zeta^{2}\right) \tau^{2} \omega^{2}-36 \pm 24 \zeta-4 \zeta^{2}}{\left(4 \mp 4 \zeta+\zeta^{2}\right) \tau^{2} \omega^{2}+36 \mp 24 \zeta+4 \zeta^{2}} \\
\sin (\omega \tau)=\frac{-(2 \mp \zeta) \omega^{3} \tau^{3}+\left(36 \mp 24 \zeta+4 \zeta^{2}\right) \omega \tau}{\left(4 \mp 4 \zeta+\zeta^{2}\right) \tau^{2} \omega^{2}+36 \mp 24 \zeta+4 \zeta^{2}}
\end{array}\right.
$$

Using the standard trigonometric identity $\cos ^{2}(\omega \tau)+\sin ^{2}(\omega \tau)=1$, we show that no positive solution exists for both cases $\left(\star_{ \pm}\right)$. As a result, under Stepan-Hassard notations (see Appendix), we obtain $K=\kappa=3$. 
Positive real roots $\omega_{k}$ of $\mathcal{R}$ and the associated $\operatorname{sign}\left(\mathcal{S}\left(\omega_{k}\right)\right)$ : Let us explore the positive roots of $\mathcal{R}$. Let $\omega>0$ be a root of the first transcendental function of (34), that is

$$
\frac{(6 \mp 2 \zeta) \cos (\omega \tau)}{\tau^{2}}+\frac{(2 \mp \zeta) \omega \sin (\omega \tau)}{\tau}+\omega^{2}-\frac{6 \mp 2 \zeta}{\tau^{2}}=0 .
$$

Multiplying the last equation by $\tau^{2}$ reduces the problem to the search of zeros of an univariate function in the variable $\rho=\omega \tau$, i.e., $F_{ \pm}(\rho)=\rho^{2}+(6 \mp 2 \zeta)(\cos (\rho)-1)+(2 \mp \zeta) \rho \sin (\rho)$. In the same fashion, we easily transform the function $S$ into the function $G_{ \pm}(\rho)=(2 \mp \zeta) \rho \cos (\rho)-(6 \mp 2 \zeta) \sin (\rho)+\rho(4 \mp \zeta)$. Hence, the vanishing of $F_{ \pm}$ enables the substitution of $\zeta$ as a function of $\rho$ :

$$
\zeta= \pm \frac{F(\rho)}{2(\cos (\rho)-1)+\rho \sin (\rho)},
$$

where $F$ is the function established in (28) and studied in Lemma 1.

In the case where $\left(\star_{+}\right)$is satisfied and using Lemma 2, we assert that the only intervals of interest for the product $\omega \tau=\rho$ are $\left.\mathcal{I}_{0}=\right] 0, \rho^{\star}\left[\right.$ and $\left.\mathcal{I}_{k}=\right] 2 k \pi, \nu_{k}\left[\left(k \in \mathbb{N}^{*}\right)\right.$. More precisely, for a given $\left.\zeta^{*} \in\right] 0, \underline{\zeta}_{0}$ [ corresponds a unique delay $\tau^{*}>0$ and a unique frequency $\left.\omega^{*} \in\right] 0, \frac{\rho^{*}}{\tau^{*}}\left[\right.$ such that $\mathcal{R}\left(\omega^{*}\right)=0$ and $\mathcal{S}\left(\omega^{*}\right)>0$. Furthermore, for a given $\left.\zeta \in\right] \underline{\zeta}_{0}, \underline{\zeta}_{1}[$, equation $\mathcal{R}(\omega)=0$ has no solution. Finally, for every $\left.\zeta^{*} \in\right] \underline{\zeta}_{k}, \underline{\zeta}_{k+1}\left[(k \geq 1)\right.$ corresponds a unique $\tau^{*}$ and a unique pair of solutions $\omega_{l, *}$ and $\omega^{l, *}$ in every interval $] \frac{2 l \pi}{\tau^{*}}, \frac{\nu_{l}}{\tau^{*}}\left[(1 \leq l \leq k)\right.$ such that $\mathcal{R}\left(\omega_{l, *}\right)=\mathcal{R}\left(\omega^{l, *}\right)=0, \mathcal{S}\left(\omega_{l, *}\right)<0$ and $\mathcal{S}\left(\omega^{l, *}\right)<0$.

In the case where $\left(\star_{-}\right)$is satisfied and using Lemma 3 and its proof, we assert that the only intervals of interest for the product $\omega \tau=\rho$ are $\left.\mathcal{J}_{0}=\right] \rho^{\star}, 2 \pi\left[\right.$ and $\left.\mathcal{J}_{k}=\right] \nu_{k}, 2(k+1) \pi\left[\left(k \in \mathbb{N}^{*}\right)\right.$ where $\nu_{k}$ is introduced in the proof of Lemma 2. Namely, for a given $\zeta^{*} \in \mathbb{R}_{+}^{*}$ there exists a unique delay $\tau^{*}>0$ and a unique frequency $\left.\omega_{0} \in\right] \frac{\rho^{*}}{\tau^{*}}, \frac{2 \pi}{\tau^{*}}[$ satisfying $\mathcal{S}\left(\omega_{0}\right)>0$, and a unique pair of solutions $\omega_{l, *}$ and $\omega^{l, *}$ in every interval $] \frac{\nu_{l}}{\tau^{*}}, \frac{2(l+1) \pi}{\tau^{*}}[(1 \leq l \leq k)$ such that $\mathcal{R}\left(\omega_{l, *}\right)=\mathcal{R}\left(\omega^{l, *}\right)=0, \mathcal{S}\left(\omega_{l, *}\right)>0$ and $\mathcal{S}\left(\omega^{l, *}\right)>0$.

Counting the number of roots dominating $s_{+}$: We apply formula (29) to deduce the number of unstable roots for (33) (see Appendix) with $n=2$ and $K=\kappa=3$, where $n$ is the order of the system, $\kappa$ is the multiplicity of 0 as a root of (33), $K$ is the total number of roots of (33) on the imaginary axis, $r$ is the number of positive real roots of $\mathcal{R}$ which is specified hereafter with respect to the two cases $\left(\star_{ \pm}\right)$.

$\left(\star_{+}\right)$In this case, $\mathcal{S}^{(3)}(0)=\zeta \tau>0$ and three different configurations are possible for $r$. Namely, $r \in\{0,1,2 k\}$ $\left(k \in \mathbb{N}^{*}\right)$. In all cases formula (29) allows to conclude that $\mathcal{Z}=0$, showing the dominancy of 0 as a root of (33) which is equivalent to the dominancy of $s_{+}$as a root of (32);

$\left(\star_{-}\right)$In this case, $\mathcal{S}^{(3)}(0)=-\zeta \tau<0$ and $r$ is an odd number. Formula (29) allows to conclude that $\mathcal{Z}=1$, entailing that 0 as a root of (33) is dominated by a single root, which is equivalent to $s_{-}$as a root of (32) being dominated by a single, necessarily real, root $s_{0}$ (the roots of quasipolynomials with real coefficients are complex conjugates).

iv). The triple root at $s_{+}$is negative if, and only if, $\sqrt{8+\tau^{2}\left(a_{1}^{2}-4 a_{0}\right)}<\tau a_{1}+4$, which reduces to $\tau^{2} a_{0}+2 \tau a_{1}+$ $2>0$. The sign of a quadratic polynomial is related to its roots $\tau_{ \pm}=\frac{-a_{1} \pm \sqrt{\Delta+2 a_{0}}}{a_{0}}=\frac{-a_{1} \pm \sqrt{a_{1}^{2}-2 a_{0}}}{a_{0}}$. Two cases are of interest here:

- If $a_{0}<0$, then $\tau_{+}>0$ and admissible delays $\tau$ lie in $] 0, \tau_{-}[$;

- If $a_{0}>0$, then $a_{1}$ has to be negative and admissible delays $\left.\tau \in\right] 0, \tau_{-}[\cup] \tau_{+}, \infty[$.

\section{ON SENSITIVITY OF THE DESIGN TO PARAMETERS VARIATION}

In the presence of parameter uncertainties, the following result from [32] emphasizes the behavior of the spectral abscissa, which is in our case a multiple root of the characteristic quasipolynomial function, with respect to parameters' variation. Accordingly, the characteristic matrix (3) is perturbed through some parameter $\epsilon$ yielding the following 
characteristic equation :

$$
\operatorname{det} \mathbb{M}(s, \tau, \epsilon)=0 .
$$

Notice that, when $\epsilon=0$ one has $\mathbb{M}(s, \tau, 0)=\mathbb{M}(s, \tau)$. Here, we focus on perturbations of a multiple eigenvalue $s_{0}$ of (3) with algebraic multiplicity $m$ and geometric multiplicity one. Define the vectors $H_{0}, \ldots H_{m-1}$ satisfying

$$
H_{0} \neq 0, \quad \sum_{k=0}^{l} \frac{1}{k !} \frac{\partial^{k} \mathbb{M}}{\partial s^{k}}\left(s_{0}, \tau\right) H_{l-k}=0, \quad l=0, \ldots, m-1,
$$

thereby forming the corresponding Jordan chain. In what follows we use subscripts for partial derivatives $\left(M_{s}:=\right.$ $\left.\frac{\partial \mathbb{M}}{\partial s}, \mathbb{M}_{\epsilon}=: \frac{\partial \mathbb{M}}{\partial \epsilon}\right)$, and to simplify the notation, we omit the argument $\left(s_{0}, \tau\right)$ when it is clear from the context.

Theorem 5.1 ([32]). Let $s_{0}$ be a spectral value of the quasipolynomial (4) for $\epsilon=0$, with algebraic multiplicity equal to $m$ and geometric multiplicity one, with Jordan chain $\left(H_{0}, \ldots, H_{m-1}\right)$. Let $U_{0}$ be the corresponding left eigenvector. Assume that condition

$$
U_{0}^{*} M_{\epsilon} H_{0} \neq 0
$$

holds. Then, around $\epsilon=0$, the eigenvalues in the vicinity of $s_{0}$ can be expanded as the branches of the Puieseux series

$$
s(\epsilon)=s_{0}+\sum_{i=1}^{\infty} \epsilon^{\frac{i}{m}} s_{i},
$$

where

$$
s_{1}^{m}=-\frac{U_{0}^{*} \mathbb{M}_{\epsilon} H_{0}}{U_{0}^{*}\left(\frac{1}{1 !} \mathbb{M}_{s} H_{m-1}+\frac{1}{2 !} \mathbb{M}_{s^{2}} H_{m-2}+\cdots+\frac{1}{m !} M_{s^{m}} H_{0}\right)} .
$$

A numerical example based on the above result is provided in the next illustrative section.

\section{ILLUSTRATIVE EXAMPLES}

In this section we exhibit the use of the MID property in control design. Indeed, results from Theorems 4.1-4.2 are exploited in tuning stabilizing controllers parameters. Discussions and interpretations follow each case study.

\subsection{Stabilizing the Double Integrator via a Delayed-Feedback controller}

Let us revisit again the problem of stabilization of the double integrator pointed out in section 3.2. Conforming with the presented approach, we consider the control problem $\ddot{\xi}(t)=u(t)$ where the controller structure is given by $u(t)=-\alpha_{0} \xi(t-\tau)-\alpha_{1} \dot{\xi}(t-\tau)$. So that, the characteristic equation corresponding to the closed-loop system is:

$$
Q(s, \tau)=s^{2}+\left(\alpha_{1} s+\alpha_{0}\right) e^{-\tau s}=0 .
$$

Note that the inclusion of the gain $\alpha_{1} \neq 0$ in the control structure increases the degree of the resulting quasipolynomial to 4. Applying the result of Theorem 4.2 from Section 4, we assign a dominant negative spectral value with multiplicity 3 at $s_{+}=\frac{-2+\sqrt{2}}{\tau}$ (corresponding to the dominant decay rate of the solution) when $\alpha_{0}$ and $\alpha_{1}$ are chosen such that:

$$
\alpha_{0}=2 \frac{(-7+5 \sqrt{2}) \mathrm{e}^{-2+\sqrt{2}}}{\tau^{2}} \text { and } \quad \alpha_{1}=2 \frac{(\sqrt{2}-1) \mathrm{e}^{-2+\sqrt{2}}}{\tau} .
$$

Observe that decreasing the delay value improves the exponential decay rate of the time-domain solution. 


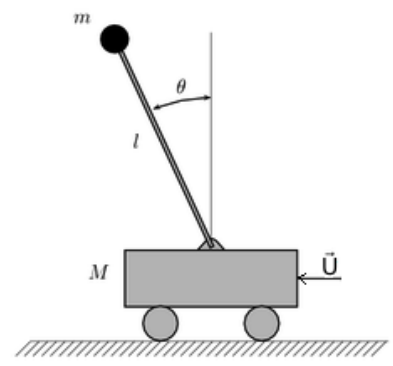

FiguRE 7. Inverted Pendulum on a cart

\subsection{Stabilization of an Inverted Pendulum on a Cart}

In the sequel we consider the friction free model presented in [51] by adopting the same notations. Denote the mass of the cart $M$, the mass of the pendulum $m$ and let the relative mass be $\epsilon=m /(m+M)$. In the dimensionless form, by neglecting the frictions, the dynamics of the inverted pendulum on a cart in Figure 7 are governed by the following ODE (see also $[6,26,52])$ :

$$
\left(1-\frac{3 \epsilon}{4} \cos ^{2}(\theta)\right) \ddot{\theta}+\frac{3 \epsilon}{8} \dot{\theta}^{2} \sin (2 \theta)-\sin (\theta)+U \cos (\theta)=0,
$$

where $U$ represents the horizontal driving force exerted by the control law which is designed as a delayed state-feedback:

$$
U(t)=\left(1-\frac{3}{4} \epsilon\right)\left(\alpha_{0} \theta(t-\tau)+\alpha_{1} \dot{\theta}(t-\tau)\right) .
$$

Hence, equation (41) can be written in closed-loop as a Delay-Differential Equation (DDE) of the form:

$$
\dot{\xi}=f(\xi(t), \xi(t-\tau)),
$$

where $\xi=\left(\xi_{1}, \xi_{2}\right)^{T}=(\theta(t), \dot{\theta}(t))^{T}$. The right hand side $f: \mathbb{R}^{2} \times \mathbb{R}^{2} \rightarrow \mathbb{R}^{2}$ reads as:

$$
f_{1}(\xi, \varsigma)=\xi_{2} \quad \text { and } \quad f_{2}(\xi, \varsigma)=\frac{-\frac{3}{8} \epsilon \sin \left(2 \xi_{1}\right) \xi_{2}^{2}+\sin \left(\xi_{1}\right)-\cos \left(\xi_{1}\right)\left(1-\frac{3}{4} \epsilon\right)\left(\alpha_{0} \varsigma_{1}+\alpha_{1} \varsigma_{2}\right)}{1-\frac{3}{4} \epsilon \cos ^{2}\left(\xi_{1}\right)}
$$

where $\varsigma(t)=\xi(t-\tau)$. The phase space of (42)-(43) is the space of continuous functions over the delay interval $[-\tau, 0]$ with values in $\mathbb{R}^{2}$. The linearization of $f$ with respect to its two arguments $\xi$ and $\varsigma$ at the origin is given by:

$$
\partial_{1} f(0,0)=\left[\begin{array}{cc}
0 & 1 \\
-a_{0} & 0
\end{array}\right] \text { and } \partial_{2} f(0,0)=\left[\begin{array}{cc}
0 & 0 \\
-\alpha_{0} & -\alpha_{1}
\end{array}\right] \text {, }
$$

where $a_{0}=\frac{1}{\frac{3}{4} \epsilon-1}$. Thus, the characteristic function is given by $Q(s, \tau)=s^{2}+a_{0}+\left(\alpha_{0}+\alpha_{1} s\right) \mathrm{e}^{-s \tau}$. Next, Theorem 4.2 enables the tuning of the controller gains as follows:

$$
\alpha_{0}=2 \frac{\left(-7+5 \sqrt{2-\tau^{2} a_{0}}+\tau^{2} a_{0}\right) \mathrm{e}^{-2+\sqrt{2-\tau^{2} a_{0}}}}{\tau^{2}} \quad \text { and } \quad \alpha_{1}=2 \frac{\left(-1+\sqrt{2-\tau^{2} a_{0}}\right) \mathrm{e}^{-2+\sqrt{2-\tau^{2} a_{0}}}}{\tau}
$$

to assign the rightmost root (a triple root) at: $s=\frac{\left(-2+\sqrt{2} \sqrt{\frac{3 \epsilon-4-2 \tau^{2}}{3 \epsilon-4}}\right)}{\tau}$. Finally, the left-free delay is to be chosen $\tau \in] 0, \tau_{-}\left[\right.$where $\tau_{-}$is defined in (31). 


\subsection{A third-order Mach number regulation in a wind tunnel model}

Transonic flows analysis is still a challenging problem in compressible fluid dynamics. In a stationary transonic flow, subsonic and supersonic regions often coexist and are respectively governed by elliptical and hyperbolic equations. Whatismore, these two types of partial differential equations require completely different approaches, a fact that usually precludes admissible solutions in the entire region. In particular, the Mach number regulation in a wind tunnel is based on the Navier-Stokes equations for unsteady flow and regulated by control laws acting on temperature and pressure.

The following simplified model of Mach number regulation described in [29] consists of a system of three state equations with a delay in one of the state variables. It is stressed that in steady-state operating conditions, the dynamic response of the Mach number perturbations $\xi_{1}$ to small perturbations in the guide vane angle actuator $\xi_{2}$ are governed by the system of equations:

$$
\left\{\begin{array}{l}
\dot{\xi}_{1}(t)=-a \xi_{1}(t)+k a \xi_{2}(t-\tau) \\
\dot{\xi}_{2}(t)=\xi_{3}(t) \\
\dot{\xi}_{3}(t)=-\omega^{2} \xi_{2}(t)-2 \eta \omega \xi_{3}(t)+\omega^{2} u(t),
\end{array}\right.
$$

where $a, \omega, \eta, k$ and $\tau$ are positive parameters depending on the operating point and presumed constant when the perturbations $\xi_{i}$ are small.

In [29], a feedback consisting of a linear combination of state variables and weighted integrals of some of the state variables over a period equal to the time delay is proposed, by which the spectrum of the closed-loop system becomes finite and arbitrarily assigned (a spectrum consisting of three negative eigenvalues). That said, our method does not render the closed-loop system finite dimensional but only involves controlling its rightmost root. Furthermore, the objective of this study is to generate reduced complexity controllers assigning an admissible decay rate of the time-domain trivial solution.

Let us consider the control law:

$$
u(t)=-\frac{\gamma}{\omega^{2}} \xi_{2}(t)-\frac{\alpha_{0}}{\omega^{2}} \xi_{2}(t-\tau)-\frac{\alpha_{1}}{\omega^{2}} \xi_{3}(t-\tau) .
$$

Accordingly, the quasipolynomial function corresponding to the closed-loop system is given by:

$$
\Delta(s, \tau)=(s+a)\left(\left(s \alpha_{1}+\alpha_{0}\right) \mathrm{e}^{-s \tau}+s^{2}+2 s \eta \omega+\omega^{2}+\gamma\right) .
$$

Since $a$ is a positive parameter, our aim is to determine conditions on the remaining parameters of the system assigning a negative rightmost root of the second factor of (46). Moreover, since the controller action does not affect the spectral value at $s=-a$, it suffices to assign the multiple root of the second factor to the left of $s=-a$ to minimize the spectral abscissa. By setting $\omega^{2}+\gamma=a_{0}$ and $2 \eta \omega=a_{1}$, the second factor of (46) takes the form (4). Therefore, one can either use Theorem 4.1 to assign a quadruple rightmost root at $s=s_{0}$ or use Theorem 4.2 to assign a triple rightmost root at $s=s_{+}$.

Keeping in mind that the delay is imposed by the model and that the discriminant is $\Delta=4\left(\omega^{2}\left(\eta^{2}-1\right)-\gamma\right)$, formula (23) from Theorem 4.1 requires the discriminant to satisfy $\Delta=-2 / \tau^{2}$. Thus, one should tune $\gamma=\omega^{2}\left(\eta^{2}-1\right)+2 / \tau^{2}$ insuring the negativity of the discriminant $\Delta$ since $\gamma>\omega^{2}\left(\eta^{2}-1\right)$. Lastly, the gains $\alpha_{0}$ and $\alpha_{1}$ are tuned according to formula (23).

Alternatively, for an applicative reason it may be more appropriate to design a stabilizing controller according to Theorem 4.2 assigning a triple negative rightmost root at $s_{+}$presented in formula $\left(\star_{+}\right)$. Since $a_{1}>0(\omega>0$ and $\eta>0)$, we first set $\gamma$ so that $s_{+}<0$, i.e., one has to set $a_{0}<0$ and $\left.\tau \in\right] 0, \tau_{-}[$as emphasized in formula (31). Once $\gamma$ is chosen, we apply ( $\left.\star_{+}\right)$to tune the gains $\alpha_{0}$ and $\alpha_{1}$.

Let us illustrate the sensitivity analysis proposed in Section 5 of the above design (45) where the gains are tuned following Theorem 4.1. Namely, we assume that the damping factor $\eta$ is subject to uncertainties. The perturbed characteristic 
matrix corresponding to the closed-loop system (44) is given by

$$
\mathbb{M}(s, \tau ; \epsilon)=\left[\begin{array}{ccc}
s+a & -\mathrm{e}^{-s \tau} k a & 0 \\
0 & s & -1 \\
0 & \omega^{2}+\gamma+\mathrm{e}^{-s \tau} \alpha_{0} & s+2 \omega(\eta+\epsilon)+\alpha_{1} \mathrm{e}^{-s \tau}
\end{array}\right]
$$

For $\epsilon=0$, a quadruple real root $s_{0}=-\frac{2+\tau \eta \omega}{\tau}$ (described in Theorem 4.1) occurs when the gains $\alpha_{0}$ and $\alpha_{1}$ satisfy:

$$
\alpha_{0}=\frac{-10-2 \tau \eta \omega}{\tau^{2} \mathrm{e}^{2+\tau \eta \omega}}, \quad \text { and } \quad \alpha_{1}=\frac{-2}{\tau \mathrm{e}^{2+\tau \eta \omega}} .
$$

By setting the parameters $\tau, \omega$ and the nominal damping factor $\eta$ according to the following table:

\begin{tabular}{|c|c|c|c|}
\hline Parameter & $\omega$ & $\eta$ & $\tau$ \\
\hline Value & 2 & $1 / 2$ & 1 \\
\hline
\end{tabular}

which guarantees $s=-3$ as a spectral abscissa of the closed-loop system, the perturbation of the damping term splits the spectral abscissa into four distinct spectral values. More precisely, if $\epsilon>0$ then it splits into two pairs of conjugate complex roots. However, if $\epsilon<0$ then $s=-3$ splits into a pair of complex roots and two other roots evolve on the real axis, see Figure 8 . In addition, the first destabilizing crossing occurs at a frequency $\omega^{*} \approx 1.9$ for the damping perturbation $\epsilon^{*} \approx-0.5826$.

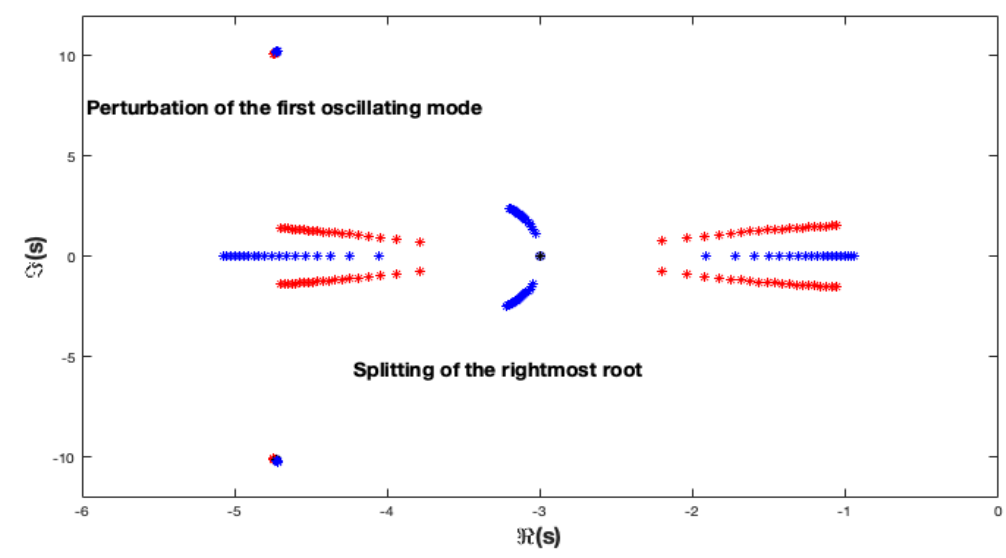

FIGURE 8. Migration of the quadruple non-semisimple spectral abscissa on the real axis, in blue the progress of $s(\epsilon)$ for $\epsilon<0$ and in red the progress of the spectral values for $\epsilon>0$. The curves are drawn for $\epsilon \in]-\bar{\epsilon}, \bar{\epsilon}$, with a step size $\varsigma=10^{-2}$ where $\bar{\epsilon}$ represents $40 \%$ of the nominal damping factor $\eta$. Notice that the perturbation $\epsilon^{*}$ has to reach $120 \%$ of the nominal value of the damping factor $\eta$ to destabilize the trivial solution. Moreover, the effect of such a perturbation is relatively insignificant on the first oscillating mode. Representative bifurcation diagram has been computed using QPmR package [57].

\section{CONCLUding REMARKS}

The Multiplicity-Induced-Dominancy is deeply investigated for second-order retarded differential equations. It seems that the sign of the discriminant of the second-order polynomial corresponding to the finite-dimensional part of the equation is involved in such a property's validity. The latter property enables a controller-design based on the trivial solution's 
decay rate assignment. Finally, to demonstrate its applicability, several case studies are numerically explored and commented.

It is worth mentioning that the inclusion of information on the delayed acceleration in the control loop yields a timedelay system of neutral type

$$
\ddot{\xi}(t)+\alpha_{2} \ddot{\xi}(t-\tau)+a_{1} \dot{\xi}(t)+\alpha_{1} \dot{\xi}(t-\tau)+a_{0} \xi(t)+\alpha_{0} \xi(t-\tau)=0 .
$$

characterized by the following quasipolynomial function of degree 5 :

$$
\Delta(s, \tau)=s^{2}+a_{1} s+a_{0}+\left(\alpha_{0}+s \alpha_{1}+s^{2} \alpha_{2}\right) e^{-\tau s} .
$$

A necessary condition for the asymptotic stability of the trivial solution of equation (48) is $\left|\alpha_{2}\right|<1$, which guarantees the stability of the corresponding difference operator, see for instance [20]. More precisely, if one assumes that $-1<\alpha_{2}<0$ then function (49) admits a negative root at $s_{0}=\frac{\ln \left(-\alpha_{2}\right)}{\tau}$ with multiplicity 5 if, and only if, the function parameters satisfy:

$$
\left\{\begin{array}{l}
\alpha_{0}=\frac{\alpha_{2}\left(12-6 \ln \left(-\alpha_{2}\right)+\left(\ln \left(-\alpha_{2}\right)\right)^{2}\right)}{\tau^{2}}, \\
\alpha_{1}=2 \frac{\alpha_{2}\left(3-\ln \left(-\alpha_{2}\right)\right)}{\tau}, \\
a_{0}=\frac{6 \ln \left(-\alpha_{2}\right)+\left(\ln \left(-\alpha_{2}\right)\right)^{2}+12}{\tau^{2}}, \\
a_{1}=2 \frac{-3-\ln \left(-\alpha_{2}\right)}{\tau} .
\end{array}\right.
$$

Furthermore, the spectrum distribution of function (49) consists of a chain of roots with real parts close to $s_{0}$. However, the analytical dominancy proof of multiple spectral values for time-delay systems of neutral type remains an open question.

\section{ACKNOWLEDGMENTS}

IB \& SIN are partially financially supported by a public grant overseen by the French National Research Agency (ANR) as part of the "Investissement d'Avenir" program, through the "iCODE Institute project" funded by the IDEX ParisSaclay and a grant from Hubert Curien (PHC) BALATON, project number 40502NM entitled "Analysis of stabilizability of delayed dynamical system as function of the systems parameters and the time delays with applications to human balancing". The authors thank Jean-Jacques Loiseau (LS2N Nantes, France) for discussions and insights on finite poleplacement for delay systems. Last but not least we thank Catherine Bonnet (Inria Saclay \& L2S) for careful reading of the manuscript and for helpful discussions.

\section{APPENDIX}

\subsection{Proofs of the technical Lemmas}

This subsection is dedicated to the proofs of Lemmas 1-3 presented in Section 2.

Proof of Lemma 1. Consider the transcendental function defined by:

$$
F(\rho)=6 \cos (\rho)+2 \rho \sin (\rho)+\rho^{2}-6,
$$

where $\rho=\omega \tau$. By continuity of $F$, one easily shows that $F(\rho)=0$ admits at least a positive root in the interval $\left.\rho^{\star} \in\right] \pi, \frac{3 \pi}{2}\left[\right.$ since $F(\pi) F\left(\frac{3 \pi}{2}\right)<0$. 
To show the uniqueness of such a solution, we first deal with the interval $[0, \pi[$. We compute the first three successive derivatives of $F$ with respect to $\rho$ :

$$
\left\{\begin{aligned}
F^{\prime}(\rho) & =-4 \sin (\rho)+2 \rho \cos (\rho)+2 \rho, \\
F^{\prime \prime}(\rho) & =-2 \cos (\rho)-2 \rho \sin (\rho)+2, \\
F^{(3)}(\rho) & =-2 \rho \cos (\rho) .
\end{aligned}\right.
$$

Obviously, $F^{(3)}$ vanishes only for $\rho \in\left\{0, \frac{\pi}{2}\right\}$, it is negative for $\left.\rho \in\right] 0, \frac{\pi}{2}[$ and positive for $\rho \in] \frac{\pi}{2}, \pi[$, thereby entailing that $F^{\prime \prime}$ is decreasing on $] 0, \frac{\pi}{2}[$ and increasing on $] \frac{\pi}{2}, \pi\left[\right.$. Then, there exists $\left.\rho_{0} \in\right] \frac{\pi}{2}, \pi\left[\right.$ such that $F^{\prime \prime}\left(\rho_{0}\right)=0$ since $F^{\prime \prime}(\pi) F^{\prime \prime}(\pi / 2)<0$. Now, for $\rho \in\left[0, \rho_{0}\right.$ [ we have $F^{\prime \prime}(\rho)<0$ and for $\left.\rho \in\right] \rho_{0}, \pi\left[\right.$ we have $F^{\prime \prime}(\rho)>0$, so that $F^{\prime}$ is decreasing on $] 0, \rho_{0}[$ and increasing on $] \rho_{0}, \pi\left[\right.$. In consequence, $F^{\prime}$ is negative on $] 0, \pi\left[\right.$ since $F^{\prime}(0)=F^{\prime}(\pi)=0$. Finally, we conclude that $F$ is negative and decreasing for $\rho \in] 0, \pi[$.

Next, for $\rho \in[\pi, 2 \pi]$ we have $F^{\prime}(\rho)>0$ so that $F$ is strictly increasing and since $F(\pi) F\left(\frac{3 \pi}{2}\right)<0$ function $F$ admits a unique zero in $] \pi, \frac{3 \pi}{2}[$.

Consider now $\rho \in[2 k \pi,(2 k+1) \pi]\left(k \in \mathbb{N}^{*}\right)$, then the following lower-bound holds for all $k \in \mathbb{N}^{*}:$

$$
\rho^{2}-6(1-\cos (\rho))+2 \rho \sin (\rho) \geq 4 \pi^{2}-6(1-\cos (\rho))+2 \rho \sin (\rho)>0
$$

and proves that $F$ has no roots in $[2 k \pi,(2 k+1) \pi]$, as illustrated by Figure 9 . Finally, consider $\rho \in[(2 k+1) \pi, 2(k+1) \pi]$,

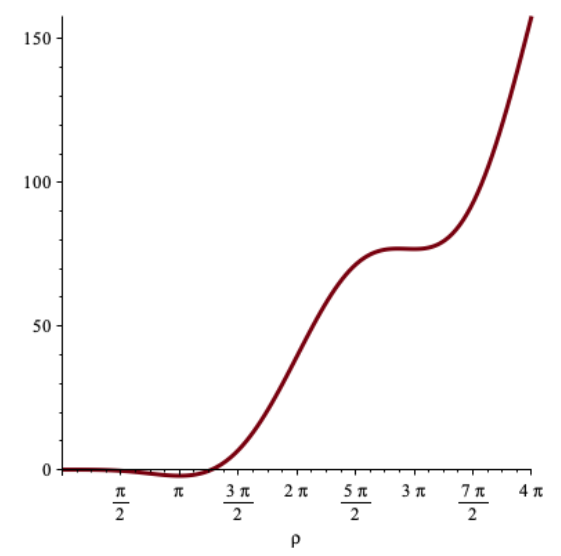

FIGURE 9. Uniqueness of the positive root of $F$ given by (50)

where $F^{\prime}(\rho)=-4 \sin (\rho)+2 \rho(1+\cos (\rho))$ is positive since $\sin (\rho)<0$. Thus, $F(\rho)$ is increasing on all intervals $\rho \in[(2 k+1) \pi,(2 k+2) \pi]$.

In conclusion, equation (50) admits a unique solution $\left.\rho^{\star} \in\right] \pi, \frac{3 \pi}{2}[$.

Proof of Lemma 2.

Analysis of $F_{+}$: The expression of $F_{+}$is linear in $\zeta$, so the vanishing of $F_{+}$allows to explicitly express $\zeta$ as a univariate function in $\rho$ :

$$
\zeta(\rho)=\frac{F(\rho)}{2(\cos (\rho)-1)+\rho \sin (\rho)}
$$

where $F$ is studied in Lemma 1; see Figure 10 illustrating the behavior of the function $\zeta(\rho)$. The analysis of the above function requires the investigation of the behavior of $D(\rho)=2(\cos (\rho)-1)+\rho \sin (\rho)$. 
First, we easily check that $D^{\prime \prime}(\rho)=-\rho \sin (\rho)=0$ if, and only if, $\rho=k \pi(k \in \mathbb{N})$. In consequence, $D^{\prime \prime}(\rho)$ has an alternating sign on successive intervals $] 2 k \pi,(2 k+1) \pi[$, it is negative on $] 0, \pi[$ then positive on $] \pi, 2 \pi[$ and so on. Thereby, $D^{\prime}(\rho)=\rho \cos (\rho)-\sin (\rho)$ is monotonic on each such interval. Moreover, one easily shows that $D^{\prime}(\rho)<0$ on the interval $] 0, \pi\left[\right.$ and vanishes at a unique $\mu_{k}$ on intervals of length $\pi$, i.e., $\left.\mu_{k} \in\right] k \pi,(k+1) \pi\left[\right.$ and $\mu_{k}$ are nothing but the fixed points of the function $\tan (\rho)$ indexed in increasing order. In turn, $D^{\prime}(\rho)$ is negative on $] 0, \mu_{1}[$ and has an alternating sign on successive intervals $] \mu_{k}, \mu_{k+1}[$. As a result, $D(\rho)$ has an alternating monotonicity; it is decreasing on ] $0, \mu_{1}[$, then increasing on $] \mu_{1}, \mu_{2}\left[\right.$ and so on. Finally, $D(2 k \pi)=D\left(\nu_{k}\right)=0$ for some $\left.\nu_{k} \in\right] \mu_{2 k},(2 k+1) \pi[(k \geq 1)$ which are nothing but the fixed points of the function $\Phi: \rho \mapsto 2(1-\cos (\rho)) / \sin (\rho)$; see the vertical red asymptotes in Figure 10.

Combining the variation of $F$ (studied in Lemma 1) with the behavior of $D$ explored above, we deduce that $\zeta(\rho)$ is strictly decreasing on $] 0,2 \pi\left[\right.$ and that for each given $\left.\zeta^{*} \in\right] 0,1\left[\right.$ there exists a unique $\rho^{*}$, root of $F_{+}$, in $] 0, \rho^{\star}[$; the behavior of $F_{+}$is depicted in Figure 10 . Since $\zeta$ has to be positive, a first conclusion is that the only intervals of interest are $] 0, \rho^{\star}[$ and $\rho \in] 2 k \pi, \nu_{k}\left[\left(k \in \mathbb{N}^{*}\right)\right.$. In addition, since $D(\rho)>0$ on $] 2 k \pi, \nu_{k}\left[\left(k \in \mathbb{N}^{*}\right)\right.$ and $\zeta$ can be written as

$$
\zeta(\rho)=2+\frac{\rho^{2}+2(\cos (\rho)-1)}{D(\rho)}>2,
$$

we deduce that $\zeta$ defines a bijection between $] 0, \rho^{\star}[$ and $] 0,1\left[\right.$. Now, regarding intervals $\in\left[2 k \pi, \nu_{k}\right]$, we know that $\zeta$ is positive and has two vertical asymptotes at $\rho=2 k \pi$ and $\rho=\nu_{k}$. So each horizontal line intersects the curve $\zeta(\rho)$ at an even number of points $\rho \in] 2 k \pi, \nu_{k}$ [ (including zero and counting multiplicity). More precisely, in order to determine the exact number of roots of $F_{+}$in such intervals, an investigation of the local minima of $\zeta$ is required. To do so, we explore the zeros of $\zeta^{\prime}$. In other words, we explore the double roots of $F_{+}$in such an interval, that is

$$
\left\{\begin{array}{l}
\zeta=\frac{6 \cos (\rho)+2 \rho \sin (\rho)+\rho^{2}-6}{2 \cos (\rho)+\rho \sin (\rho)-2}, \\
\zeta=\frac{-2 \rho+4 \sin (\rho)-2 \rho \cos (\rho)}{\sin (\rho)-\rho \cos (\rho)} .
\end{array}\right.
$$

Next, the elimination of the trigonometric functions from the last system of equations produces the following algebraic equation

$$
(-2+\zeta)^{2} \rho^{2}-48+12 \zeta^{3}-\zeta^{4}-51 \zeta^{2}+88 \zeta=0,
$$

representing a necessary condition for a root of $F_{+}$to be double and from which the unique admissible positive solution for the argument of the minimum of $\zeta$ is obtained:

$$
\rho(\zeta)=\frac{\sqrt{(\zeta-1)(\zeta-3)}(\zeta-4)}{\zeta-2} .
$$

Combining the last condition (54) with (52) yields that $\zeta$ is the fixed point of the function: $\Psi: \zeta \mapsto F_{+}(\rho(\zeta)) / D(\rho(\zeta))$. Equivalently, we draw the algebraic expression of $\zeta$ as a function of $\rho$,

$$
\left\{\begin{aligned}
\zeta(\rho)= & \frac{1}{6} \sqrt{\left(\frac{\left(-3(h(\rho))^{2}+\left(36+12 \rho^{2}\right) h(\rho)-27+18 \rho^{2}-3 \rho^{4}\right)}{h(\rho)}+\frac{36 \sqrt{3}(\rho-1)(\rho+1)}{l(\rho)}\right)} \\
& +3+\frac{\sqrt{3}}{6} l(\rho) \quad \text { where } \\
l(\rho)= & \sqrt{\frac{6 h(\rho)+2 \rho^{2} h(\rho)+(h(\rho))^{2}+r(\rho)}{\sqrt[3]{q(\rho)+12 p(\rho)}}}, h(\rho)=\sqrt[3]{q(\rho)+12 p(\rho)}, \\
p(\rho)= & \sqrt{3 \rho^{8}-27 \rho^{6}+405 \rho^{4}-81 \rho^{2}}, q(\rho)=-\rho^{6}+9 \rho^{4}-243 \rho^{2}+27, r(\rho)=\rho^{4}-6 \rho^{2}+9 .
\end{aligned}\right.
$$


In each interval $\left.\mathcal{I}_{k}=\right] 2 k \pi, \nu_{k}$ [ the function $\zeta$ is analytic, owing to its proven positivity and its behavior with respect to variations of $\rho$ as it describes a convex parabola between the two vertical asymptotes at $\rho=2 k \pi$ and $\rho=\nu_{k}$; see Figure 10 for further insights. As a matter of fact, function $\zeta(\rho)$ has a unique $\underline{\zeta}_{k}$ minimum on each interval $\mathcal{I}_{k}\left(k \in \mathbb{N}^{*}\right)$ whose argument is a unique $\left.\rho_{k} \in\right] 2 k \pi, \nu_{k}$. Therefore, each $\zeta>\underline{\zeta}_{k}$ yields two positive numbers $\underline{\rho}_{k}<\bar{\rho}_{k}$ in $\mathcal{I}_{k}$ satisfying $F_{+}\left(\underline{\rho}_{k}\right)=F_{+}\left(\bar{\rho}_{k}\right)=0$.

Analysis of $G_{+}$: Since we are dealing with the sign of $G_{+}$at a given root $\rho^{*}$ of $F_{+}$and the corresponding positive
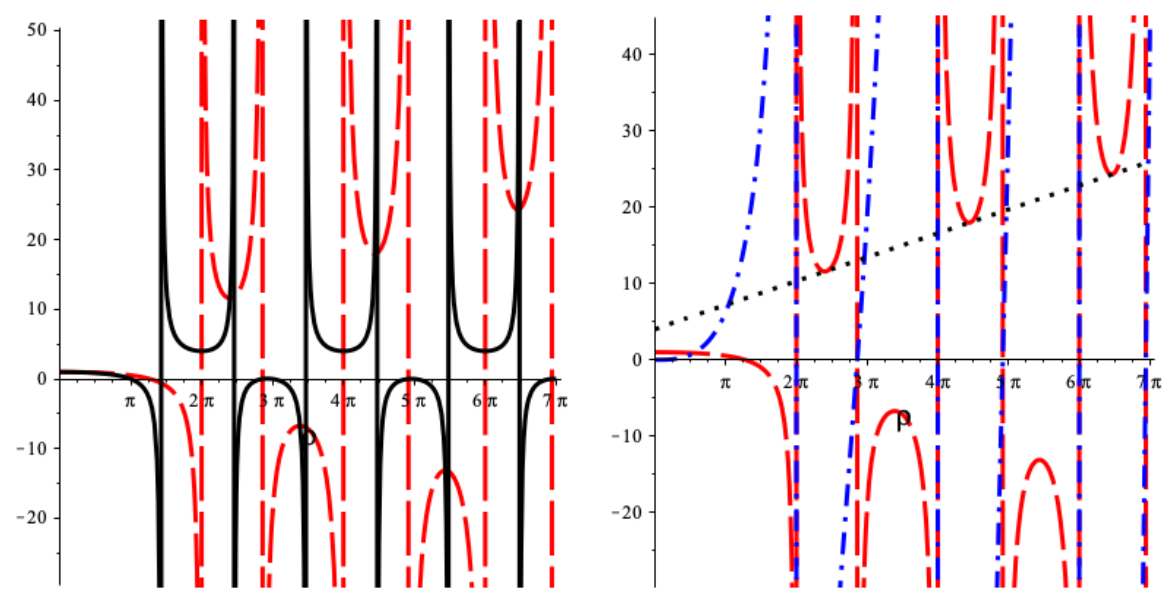

FIGURE 10. (Left panel) The dashed red curves represent the value of $\zeta$ with respect to $\rho$ such that $F_{+} \equiv 0$, the solid black curves represent the variation of $\zeta$ with respect to $\rho$ resulting from $F_{+}^{\prime} \equiv 0$ and given by (53). (Right panel) The dashed red curves represent the value of $\zeta$ with respect to $\rho$ such that $F_{+} \equiv 0$, the dash-dot blue curves correspond to $G_{+\left.\right|_{F_{+}(\rho)=0}}$, the vertical lines represent the asymptotes of both functions and the dotted black curve represents another characterization the minimum of $\zeta(\rho)$ in the intervals $\left.I_{k}=\right] 2 k \pi, \nu_{k}$ [ obtained from (55).

parameter $\zeta^{*}$, we can substitute the expression of $\zeta$ given by (52) in the expression of $G_{+}$given by (9). So that, one gets:

$$
G_{+}(\rho)_{\left.\right|_{F_{+}(\rho)=0}}=\frac{\hat{H}(\rho) \rho}{\sin (\rho)} \quad \text { where } \quad \hat{H}(\rho)=2 \sin (\rho)-(\cos (\rho)+1) \rho .
$$

Let us first explore the behavior of the function $\hat{H}$ for positive $\rho$. Since $\hat{H}^{\prime \prime}(\rho)=\rho \cos (\rho), \hat{H}^{\prime \prime}$ vanishes at $(2 k+1) \frac{\pi}{2}$ $\left(k \in \mathbb{N}^{*}\right)$. Hence, $\hat{H}^{\prime \prime}$ is positive on $] 0, \frac{\pi}{2}[$ then it has an alternating sign on successive intervals of length $\pi$; it is negative on $] \frac{\pi}{2}, \frac{3 \pi}{2}[$ then positive on $] \frac{3 \pi}{2}, \frac{5 \pi}{2}$ [ and so on. Accordingly, $\hat{H}^{\prime}$ has an alternating monotonicity and there exists $\left.\chi_{k} \in\right] \frac{\pi}{2}+2(k-1) \pi,(2 k-1) \pi\left[\left(k \in \mathbb{N}^{*}\right)\right.$ such that $\hat{H}^{\prime}$ is strictly positive on $] 2(k-1) \pi, \chi_{k}$ [ and strictly negative on ]$\chi_{k}, 2 k \pi\left[\left(k \in \mathbb{N}^{*}\right)\right.$. As a result, $\hat{H}$ exhibits an alternating monotonicity, it is strictly increasing on $\left.\rho \in\right] 2(k-1) \pi, \chi_{k}[$ and strictly decreasing on $\rho \in] \chi_{k}, 2 k \pi\left[\right.$. Moreover, $\hat{H}$ vanishes exclusively on the set $\left\{0,(2 k+1) \pi, \nu_{k}\right\}_{k \in \mathbb{N}^{*}}$ where $\nu_{k}$ are the zeros of $D$ and equivalently the fixed points of function $\Phi$ defined above. Recall that $\left.\nu_{k} \in\right] \mu_{2 k},(2 k+1) \pi[$ and $\left.\mu_{k} \in\right] k \pi,(k+1) \pi\left[\left(k \in \mathbb{N}^{*}\right)\right.$ so that $\nu_{k}$ is not a multiple of $\pi$. In conclusion, $\hat{H}$ is of alternating sign, it is strictly positive on $] 0, \pi[$, strictly negative on $](2 k-1) \pi, \nu_{k}[$ and strictly positive on $] \nu_{k},(2 k+1) \pi\left[\right.$. As such, $G_{+} \mid F_{+}=0>0$ on $] 0, \rho^{\star}[$ which completes item i).

Furthermore, we have shown that $\left.G_{+}\right|_{F_{+}=0}$ is increasing and negative on $] 2 k \pi, \nu_{k}$ [ (it has roots at $\nu_{k}$ and vertical asymptotes at $2 k \pi$ ) and that $F_{+}$admits an even number of roots (including zero and counting multiplicity) which concludes item ii). 
To summarize, for a given $\left.\zeta^{*} \in\right] 0, \underline{\zeta}_{0}\left[\left(\underline{\zeta}_{0}=1\right)\right.$ there exists a unique root $\left.\rho^{*} \in\right] 0, \rho^{\star}\left[\right.$ such that $F_{+}\left(\rho^{*}\right)=0$ and $G_{+}\left(\rho^{*}\right)>0$. Next, if $\left.\zeta^{*} \in\right] \underline{\zeta}_{0}, \underline{\zeta}_{1}\left[\right.$ no solution exists for $F_{+}(\rho)=0$. Finally, for any given $\left.\zeta^{*} \in\right] \underline{\zeta}_{k}, \infty\left[\left(k \in \mathbb{N}^{*}\right)\right.$ there exists an even number of roots in each interval $] 2 k \pi, \nu_{k}\left[\right.$ at which points $G_{+}$is negative.

Proof of Lemma 3. The proof follows the same steps and arguments as Lemma 2. Observe that the expression of $F_{-}$is also linear in $\zeta$, so the vanishing of $F_{-}$allows again to write down explicitly $\zeta$ as a univariate function in $\rho$ :

$$
\zeta(\rho)=-\frac{F(\rho)}{2(\cos (\rho)-1)+\rho \sin (\rho)}
$$

where $F$ is studied in Lemma 1 . Furthermore, one easily checks that $G_{-\left.\right|_{F_{-}}(\rho)=0}(\rho)=G_{+\left.\right|_{F_{+}}(\rho)=0}(\rho)$ which, based on the analysis carried out in the proof of Lemma 2, ends the proof; see Figure 11 for further insights.
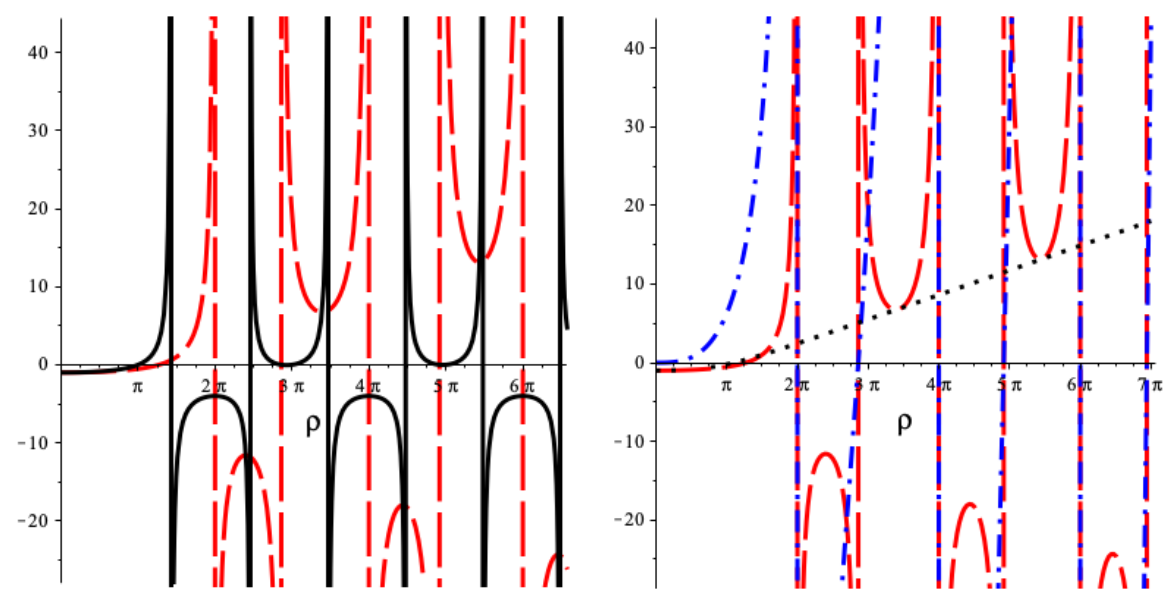

FIGURE 11. (Left panel) The dashed red curves represent the value of $\zeta$ with respect to $\rho$ given in (57) such that $F_{-} \equiv 0$ and the solid black curves represent the variation of $\zeta$ with respect to $\rho$ resulting from $F_{-}^{\prime} \equiv 0$. (Right panel) The dashed red curves represent the value of $\zeta$ with respect to $\rho$ such that $F_{-} \equiv 0$, the dash-dot blue curves represent $G_{-\left.\right|_{F_{-}(\rho)=0}}$, the vertical lines represent the asymptotes of both functions and the dotted black curve represents another characterization of the minimum of $\zeta(\rho)$ in the intervals $\left.J_{k}=\right] \nu_{k}, 2(k+1) \pi[$ obtained from (55).

\subsection{The Argument principle applied to the dominancy proof}

Here, we use the the argument principle to show the dominancy of the root $s_{0}$ of $Q$ of multiplicity 4 such that with $s_{0} \equiv x_{0} \in \mathbb{R}$. The proof is straightforward for the root of multiplicity 3 . Let $s=x+i \omega$.

Following the approach of [21,54], we shall first establish the asymptotic properties of $Q(s, \tau)$ and the corresponding logarithmic derivative $\partial_{s} Q(s, \tau) / Q(s, \tau)$. Secondly, we deflate $Q(s, \tau)$ to remove $s_{0}$ from the path $x_{0}+i \omega$ running parallel to the left of the Fourier axis. Third, we apply the argument principle to count the number of zeros (if any) in the plane $\Re(s)>s_{0}$ of the deflated function $\widetilde{Q}(s)$ and work out the asymptotic behavior of the logarithmic residue as the chosen contour is increased. In the end, the number of zeros of the deflated function in $\Re(s)>s_{0}$ coincides with that of the original characteristic function $Q(s, \tau)$ written in (4). 
Asymptotic behavior of $Q(s, \tau)$ and $\partial_{s} Q(s, \tau) / Q(s, \tau)$ : For convenience, the characteristic function defined by (4) can be rewritten in the form

$$
Q(s, \tau)=s^{2}+d_{1}(s, \tau) s+d_{2}(s, \tau)
$$

where $d_{1}(s, \tau)=a_{1}+\alpha_{1} e^{-s \tau}$ and $d_{2}(s, \tau)=a_{0}+\alpha_{0} e^{-s \tau}$. The derivative of (58) reads

$$
\partial_{s} Q(s, \tau)=\left[2+\partial_{s} d_{1}(s, \tau)\right] s+d_{1}(s, \tau)+\partial_{s} d_{2}(s, \tau),
$$

where $\partial_{s} d_{1}(s, \tau)=-\alpha_{1} \tau e^{-s \tau}$ and $d_{1}(s, \tau)+\partial_{s} d_{2}(s, \tau)=\mathcal{O}(1)$ as $|s| \rightarrow \infty$ in $\Re(s)>s_{0}$. Then,

$$
\partial_{s} Q(s, \tau)=\left[2+\partial_{s} d_{1}(s, \tau)\right] s+\mathcal{O}(1)
$$

and

$$
\frac{\partial_{s} Q(s, \tau)}{Q(s, \tau)}=s^{-1}\left[2+\partial_{s} d_{1}(s, \tau)\right]+\mathcal{O}\left(|s|^{-2}\right)
$$

as $|s| \rightarrow \infty$ in $\Re(s)>s_{0}$.

Deflation: Since $s_{0}$ is the rightmost root of multiplicity 4 of the original quasipolynomial function defined by (4), we set

$$
P(s)=\left(s-s_{0}\right)^{4} \quad \text { and } \quad \widetilde{Q}(s, \tau)=\frac{Q(s, \tau)}{P(s)}
$$

$\widetilde{Q}$ being the deflated function corresponding to $Q$ in $\Re(s)>s_{0}$. Then, $\widetilde{Q}(s, \tau)$ is holomorphic and is non zero on the path $s_{0}+i \omega$ parallel to the imaginary axis. The last statement justifies, in particular, the applicability of the argument principle in the following.

$\underline{\text { Integration contour: }}$ Let $C_{R}$ be the contour $g_{1} \cup g_{2} \cup g_{3} \cup g_{4}$ displayed in Figure 1 (right) for any $R \in \mathbb{R}$ such that

$$
\begin{aligned}
& g_{1}=\left\{s=R e^{i \theta}, \theta \in\left[-\frac{\pi}{2}, \frac{\pi}{2}\right]\right\}, \\
& g_{2}=\left\{s=-x+i R, x \in\left[0,-s_{0}\right]\right\}, \\
& g_{3}=\left\{s=s_{0}+i \omega, \omega \in[-R, R]\right\}, \\
& g_{4}=\left\{s=x-i R, x \in\left[s_{0}, 0\right]\right\} .
\end{aligned}
$$

For $R \rightarrow \infty$, all zeros of $Q(s, \tau)$ in $\Re(s)>s_{0}$ are inside $C_{R}$. By the argument principle, the number of these zeros, counted by multiplicity is

$$
\mathcal{Z}=\frac{1}{2 \pi i} \oint_{C_{R}} \frac{\partial_{s} \widetilde{Q}(s, \tau)}{\widetilde{Q}(s, \tau)} d s
$$

which can be explicitly rewritten over the Bromwich-like contour as

$$
\mathcal{Z}=\frac{1}{2 \pi i} \int_{g_{1}} \frac{\partial_{s} \widetilde{Q}(s, \tau)}{\widetilde{Q}(s, \tau)} d s+\frac{1}{2 \pi i} \int_{g_{2} \cup g_{4}} \frac{\partial_{s} \widetilde{Q}(s, \tau)}{\widetilde{Q}(s, \tau)} d s+\frac{1}{2 \pi i} \int_{g_{3}} \frac{\partial_{s} \widetilde{Q}(s, \tau)}{\widetilde{Q}(s, \tau)} d s .
$$

In the following, we evaluate the three integrals involved in (63).

Integral over $\boldsymbol{g}_{\mathbf{1}}$ and its limit as $\boldsymbol{R} \rightarrow \infty$ : For $R \gg 1, Q(s, \tau)$ and $P(s)$ are nonzero for $z$ on $g_{1}$, and

$$
\frac{1}{2 \pi i} \int_{g_{1}} \frac{\partial_{s} \widetilde{Q}(s, \tau)}{\widetilde{Q}(s, \tau)} d s=\frac{1}{2 \pi i} \int_{g_{1}} \frac{\partial_{s} Q(s, \tau)}{Q(s, \tau)} d s-\frac{1}{2 \pi i} \int_{g_{1}} \frac{P^{\prime}(s)}{P(s)} d s .
$$


Using the asymptotic behavior (60) as $|s| \rightarrow \infty$ in $\Re(s)>s_{0}$, the first integral in the r.h.s. of (64) can be rewritten as

$$
\frac{1}{2 \pi i} \int_{g_{1}} \frac{\partial_{s} Q(s, \tau)}{Q(s, \tau)} d s=\frac{1}{2 \pi} \int_{-\frac{\pi}{2}}^{\frac{\pi}{2}}\left[2+\partial_{s} d_{1}\left(R e^{i \theta}, \tau\right)\right] d \theta+\mathcal{O}\left(R^{-1}\right)
$$

In the r.h.s. integral, $\left|\partial_{s} d_{1}\left(R e^{i \theta}, \tau\right)\right| \leq \alpha_{1} \tau e^{-R \tau \cos \theta}$ is bounded and integrable and $\lim _{R \rightarrow \infty} \partial_{s} d_{1}\left(R e^{i \theta}, \tau\right)=0$ for $\tau>0$ and $\theta \in\left(-\frac{\pi}{2}, \frac{\pi}{2}\right)$. Hence, by Lebesgue's Theorem we get

$$
\lim _{R \rightarrow \infty} \frac{1}{2 \pi i} \int_{g_{1}} \frac{\partial_{s} Q(s, \tau)}{Q(s, \tau)} d s=1 .
$$

Under the same setup, the second integral in (64) reads

$$
\frac{1}{2 \pi i} \int_{g_{1}} \frac{P^{\prime}(s)}{P(s)} d s=\frac{2}{\pi} \int_{-\frac{\pi}{2}}^{\frac{\pi}{2}} \frac{d \theta}{1-\frac{s_{0}}{R} e^{-i \theta}}=2+\mathcal{O}\left(R^{-1}\right) .
$$

Thus, for $R \gg\left|s_{0}\right|$, we get

$$
\lim _{R \rightarrow \infty} \frac{1}{2 \pi i} \int_{g_{1}} \frac{P^{\prime}(s)}{P(s)} d s=\lim _{R \rightarrow \infty} \frac{2}{\pi} \int_{-\frac{\pi}{2}}^{\frac{\pi}{2}} \frac{d \theta}{1-\frac{s_{0}}{R} e^{-i \theta}}=2
$$

and therefore,

$$
\lim _{R \rightarrow \infty} \frac{1}{2 \pi i} \int_{g_{1}} \frac{\partial_{s} \widetilde{Q}(s, \tau)}{\widetilde{Q}(s, \tau)} d s=-1
$$

Following the same steps of [21], in the case of a quasipolynomial function having $\operatorname{deg}\left(P_{0}\right)=n \in \mathbb{N}$, it can be checked that the integral (65) reads,

$$
\lim _{R \rightarrow \infty} \frac{1}{2 \pi i} \int_{g_{1}} \frac{\partial_{s} Q(s, \tau)}{Q(s, \tau)} d s=\frac{n-K}{2},
$$

where $K=\kappa+2 p, \kappa$ represents the multiplicity of $s_{0}$ and $p$ the number of pairs of roots $s_{0} \pm i \omega$ running parallel to the imaginary axis. According to the proof of theorem $4.1, p=0$ and

$$
\lim _{R \rightarrow \infty} \frac{1}{2 \pi i} \int_{g_{1}} \frac{\partial_{s} Q(s, \tau)}{Q(s, \tau)} d s=\frac{n-\kappa}{2} .
$$

For $n=2$ and $\kappa=4$, the result of the integral (67) is recovered.

Integral over $\boldsymbol{g}_{\mathbf{2}} \cup \boldsymbol{g}_{\mathbf{4}}$ and its limit as $\boldsymbol{R} \rightarrow \infty$ : Once again, for $R \gg 1$, we have

$$
\frac{1}{2 \pi i} \int_{g_{2} \cup g_{4}} \frac{\partial_{s} \widetilde{Q}(s, \tau)}{\widetilde{Q}(s, \tau)} d s=\frac{1}{2 \pi i} \int_{g_{2}} \frac{\partial_{s} \widetilde{Q}(s, \tau)}{\widetilde{Q}(s, \tau)} d s+\frac{1}{2 \pi i} \int_{g_{4}} \frac{\partial_{s} \widetilde{Q}(s, \tau)}{\widetilde{Q}(s, \tau)} d s .
$$

First, we consider the first term on the r.h.s. wherein we insert the asymptotic behavior given in (60) as follows

$$
\begin{aligned}
\frac{1}{2 \pi i} \int_{g_{2}} \frac{\partial_{s} \widetilde{Q}(s, \tau)}{\widetilde{Q}(s, \tau)} d s & =\frac{1}{2 \pi i} \int_{g_{2}} \frac{\partial_{s} Q(s, \tau)}{Q(s, \tau)} d s-\frac{1}{2 \pi i} \int_{g_{2}} \frac{P^{\prime}(s)}{P(s)} d s \\
& =\frac{1}{2 \pi i} \int_{0}^{x_{0}} \frac{2+\partial_{s} d_{1}(x+i R)}{x+i R} d x-\frac{1}{2 \pi i} \int_{0}^{x_{0}} \frac{4 d x}{x-x_{0}+i R}
\end{aligned}
$$


Since

$$
\left|\frac{\partial_{s} d_{1}(x+i R)}{x+i R}\right| \leq \frac{\alpha_{1} \tau e^{-x \tau}}{\sqrt{x^{2}+R^{2}}} \quad \text { and } \quad \lim _{R \rightarrow \infty} \frac{\alpha_{1} \tau e^{-x \tau}}{\sqrt{x^{2}+R^{2}}}=0,
$$

the integral term over $\partial_{s} d_{1}(x+i R)$ vanishes asymptotically such that,

$$
\lim _{R \rightarrow \infty} \frac{1}{2 \pi i} \int_{g_{2}} \frac{\partial_{s} \widetilde{Q}(s, \tau)}{\widetilde{Q}(s, \tau)} d s=\lim _{R \rightarrow \infty} \frac{1}{\pi i} \ln \left(1-i \frac{x_{0}}{R}\right)+\lim _{R \rightarrow \infty} \frac{2}{\pi i} \ln \left(1+i \frac{x_{0}}{R}\right)=0 .
$$

Accordingly the integral over $g_{4}$ is the opposite, so that

$$
\frac{1}{2 \pi i} \int_{g_{2} \cup g_{4}} \frac{\partial_{s} \widetilde{Q}(s, \tau)}{\widetilde{Q}(s, \tau)} d s=0 .
$$

Integral over $\boldsymbol{g}_{3}$ as $\boldsymbol{R} \rightarrow \infty$ : We first determine the real and imaginary parts of the characteristic function $\widetilde{Q}(s, \tau)$ given in (58) and make use of the polar representation of an entire function. Setting $s=x_{0}+i \omega$, we introduce the real and imaginary parts of (58) as $\mathcal{R}(\omega)=\Re\left[i^{-2} Q\left(x_{0}+i \omega, \tau\right)\right]$ and $\mathcal{S}(\omega)=\Im\left[i^{-2} Q\left(x_{0}+i \omega, \tau\right)\right]$ so that the original quasipolynomial defined by (4) is rewritten as $Q(s, \tau)=\mathcal{R}(\omega)+i \mathcal{S}(\omega)$. According to (61),

$$
\widetilde{Q}(s, \tau)=\frac{Q(s, \tau)}{\left(s-s_{0}\right)^{4}}
$$

which leads to

$$
M(\omega)+i N(\omega)=\frac{\mathcal{R}(\omega)+i \mathcal{S}(\omega)}{\omega^{4}}
$$

where $M(\omega)=\Re\left[i^{2} \widetilde{Q}\left(x_{0}+i \omega, \tau\right)\right]$ and $N(\omega)=\Im\left[i^{2} \widetilde{Q}\left(x_{0}+i \omega, \tau\right)\right]$. More precisely, one gets

$$
\left\{\begin{array}{l}
M(\omega)=\frac{1}{\omega^{4}}\left\{\omega^{2}-\left(\alpha_{0}+x_{0} \alpha_{1}\right) e^{-x_{0} \tau} \cos (\tau \omega)-\alpha_{1} e^{-x_{0} \tau} \omega \sin (\tau \omega)-x_{0}^{2}-a_{1} x_{0}-a_{0}\right\} \\
N(\omega)=\frac{1}{\omega^{4}}\left\{-\left(a_{1}+2 x_{0}\right) \omega-\alpha_{1} e^{-x_{0} \tau} \omega \cos (\tau \omega)+\left(\alpha_{0}+x_{0} \alpha_{1}\right) e^{-x_{0} \tau} \sin (\tau \omega)\right\}
\end{array}\right.
$$

Note that function $M$ is even and function $N$ is odd, hence the polar representation $M(\omega)+i N(\omega)=A(\omega) e^{i \phi(\omega, \tau)}$, where

$$
A(\omega)=\sqrt{M^{2}(\omega)+N^{2}(\omega)} \text { and } \phi(\omega)=\arctan \frac{N(\omega)}{M(\omega)} .
$$

which enables us to rewrite the logarithmic derivative of $\widetilde{Q}(s, \tau)$ in this fashion

$$
\frac{\partial_{s} \widetilde{Q}(s, \tau)}{\widetilde{Q}(s, \tau)}=-i \frac{\partial_{s} A(\omega)}{A(\omega)}+\partial_{s} \phi(\omega)
$$

and accordingly

$$
\frac{1}{2 \pi i} \int_{g_{3}} \frac{\partial_{s} \widetilde{Q}(s, \tau)}{\widetilde{Q}(s, \tau)} d s=\frac{1}{2 \pi i} \int_{R}^{-R}\left[\frac{\partial_{s} A(\omega)}{A(\omega)}+i \partial_{s} \phi(\omega)\right] d \omega
$$

Since $A(\omega)$ is even, $\partial_{s} A(\omega)$ is odd and $\partial_{s} A(\omega) / A(\omega)$ is odd thereby. As a result

$$
\frac{1}{2 \pi i} \int_{g_{3}} \frac{\partial_{s} \widetilde{Q}(s, \tau)}{\widetilde{Q}(s, \tau)} d s=\frac{1}{\pi}[\phi(0)-\phi(R)]
$$


From (71), for $\omega \gg 1$ we have $\frac{N(\omega)}{M(\omega)} \sim \frac{1}{\omega}$ such that $\lim _{R \rightarrow \infty} \phi(R)=0$ and

$$
\lim _{R \rightarrow \infty} \frac{1}{2 \pi i} \int_{g_{3}} \frac{\partial_{s} \widetilde{Q}(s, \tau)}{\widetilde{Q}(s, \tau)} d s=\frac{\phi(0)}{\pi} .
$$

Determination of $\phi(0)$ : Taking into account that $\phi(\omega)=\arctan \frac{N(\omega)}{M(\omega)}$, we should first assess $M(0)$ and $N(0)$. Since the original deflated $\widetilde{Q}(s, \tau) \neq 0$ inside $C_{R}$ and $N(\omega)$ is odd, $N(0)=0$ and the sign of $M(0)$ is to be investigated. Using the following relations

$$
x_{0}^{2}+a_{1} x_{0}+a_{0}=-\frac{3}{4} \delta, a_{1}+2 x_{0}=-\sqrt{-2 \delta}, \alpha_{1} e^{-x_{0} \tau}=-\frac{1}{2} \sqrt{-2 \delta},\left(\alpha_{0}+\alpha_{1} x_{0}\right) e^{-x_{0} \tau}=\frac{3}{4} \delta
$$

where $\delta=a_{1}^{2}-4 a_{0}$, we rewrite (71) in the compact form

$$
\left\{\begin{array}{l}
M(\omega)=\frac{1}{\omega^{4}}\left[\omega^{2}-\frac{3}{4} \delta \cos (\tau \omega)+\frac{1}{2} \sqrt{-2 \delta} \omega \sin (\tau \omega)+\frac{3}{4} \delta\right] \\
N(\omega)=\frac{1}{\omega^{4}}\left[\sqrt{-2 \delta} \omega+\frac{1}{2} \sqrt{-2 \delta} \omega \cos (\tau \omega)-\frac{3}{4} \delta \sin (\tau \omega)\right] .
\end{array}\right.
$$

Both functions present the indermination $0 / 0$ in the limit $\omega \rightarrow 0$. L'Hospital's rule for the given multiplicity then yields

$$
\left\{\begin{array}{l}
M(0)=\lim _{\omega \rightarrow 0} \frac{1}{4 !}\left[\frac{1}{2} \sqrt{-2 \delta} \tau^{3}(\omega \sin (\tau \omega)-4 \cos (\tau \omega))-\frac{3}{4} \delta \tau^{4} \cos (\tau \omega)\right]=\frac{2}{3 \delta}<0 \\
N(0)=\lim _{\omega \rightarrow 0} \frac{1}{4 !}\left[\frac{1}{2} \sqrt{-2 \delta} \tau^{3}(4 \sin (\tau \omega)+\tau \omega \cos (\tau \omega))-\frac{3}{4} \delta \tau^{4} \sin (\tau \omega)\right]=0
\end{array}\right.
$$

which shows that $\mathcal{S}^{(4)}(0)=0$ and that $\widetilde{Q}(\omega, \tau)$ is continuous for $\omega=0$ as expected. Hence, $\lim _{\omega \rightarrow 0} \phi(\omega)=\pi$ and finally,

$$
\lim _{R \rightarrow \infty} \frac{1}{2 \pi i} \int_{g_{3}} \frac{\partial_{s} \widetilde{Q}(s, \tau)}{\widetilde{Q}(s, \tau)} d s=1 .
$$

Though this result may be right at first glance, one should investigate whether the quasipolynomial $M(\omega)$ has other zeros beyond $x_{0}$ for different values of $\tau$.

According to the proof of theorem 4.1, $M(\omega)$ has an only one positive zero $\left.\rho^{\star}=\tau^{*} \omega^{*} \in\right] \pi, \frac{3 \pi}{2}[$. The curve $M(\omega)+i N(\omega)$ for $0 \leq \omega \leq \omega^{*}$ starts at $M(0)=\frac{2}{3 \Delta}<0$ satisfying the condition d) of [21] (i.e. page 228) and evolves on a path in the halfplane $N(\omega)>0$. Furthermore, the curve is constrained not to intersect the origin $(0 ; 0)$ but to wing around it. This statement follows from the fact that $\tilde{Q}(0, \tau)=M(0)+i N(0) \neq 0$ at the origin. In this case, the change in $\phi(\omega)$ on $\left[0, \omega^{*}\right]$ is given by

$$
\phi\left(\omega^{*}\right)-\phi(0)=\frac{\pi}{2} \operatorname{sgn} N\left(\omega^{*}\right) \operatorname{sgn} M\left(\frac{\omega^{*}}{2}\right),
$$

where the choice $\omega^{*} / 2$ on the argument of $M\left(\omega^{*} / 2\right)$ is based on the intermediate value theorem. Now we evaluate the change in $\phi(\omega)$ on $\left[\omega^{*}, \infty\right)$. The function $M(\omega)+i N(\omega)$ starts at $i N\left(\omega^{*}\right)$ and remains for $\omega>\omega^{*}$ in the halfplane $M>0$. Hence,

$$
0-\phi\left(\omega^{*}\right)=-\frac{\pi}{2} \operatorname{sgn} N\left(\omega^{*}\right)
$$

such that, resuming (74) and (75), the full change of $\phi(\omega)$ in the interval $[0, \infty)=\left[0, \omega_{1}\right] \cup\left[\omega_{1}, \infty\right)$ reads,

$$
0-\phi(\omega)+\phi(\omega)-\phi(0)=0-\phi(0)=\frac{\pi}{2} \operatorname{sgn} N\left(\omega^{*}\right) \operatorname{sgn} M\left(\frac{\omega^{*}}{2}\right)-\frac{\pi}{2} \operatorname{sgn} N\left(\omega^{*}\right) .
$$


and

$$
\frac{\phi(0)}{\pi}=\frac{1}{2} \operatorname{sgn} N\left(\omega^{*}\right)-\frac{1}{2} \operatorname{sgn} N\left(\omega^{*}\right) \operatorname{sgn} M\left(\frac{\omega^{*}}{2}\right) .
$$

According to the proof of theorem 4.1, in the last formula, $\operatorname{sgn} M\left(\omega^{*} / 2\right)=-1$ and $\operatorname{sgn} N\left(\omega^{*}\right)=1$ for $\omega \in\left[\frac{\pi}{\tau^{*}}, \frac{3 \pi}{2 \tau^{*}}\right]$ such that $\frac{\phi(0)}{\pi}=\frac{1}{2} \cdot 1-\frac{1}{2}(1) \cdot(-1)=1$.

A consistency cross-check: As a consistency cross-check, we make use of the formula given in [21, 54], see also (29):

$$
\frac{\phi(0)}{\pi}=\frac{1}{2}(-1)^{r} \operatorname{sgn} \mathcal{S}^{(4)}(0)+\sum_{i=1}^{r}(-1)^{i-1} \operatorname{sgn} \mathcal{S}\left(\omega_{i}\right) .
$$

where, $\left\{\omega_{1} \ldots \omega_{r}\right\}$ is the set of roots of the original quasipolynomial $Q(\omega, \tau)$, such that $x_{0}<\omega_{r} \leq \ldots \leq \omega_{1}$ with $r=m+p$. Here, $m$ corresponds to the total number of positive zeros of the original function $\mathcal{R}(\omega)=M(\omega) \omega^{4}$ counted by multiplicity and $p$ represents the number of pairs of roots $\left\{s_{0} \pm i \eta_{1}, \ldots s_{0} \pm i \eta_{p}\right\}$ of $Q(s, \tau)$ which are parallel to the imaginary axis. According to the same proof 4.1, $\operatorname{sgn} \mathcal{S}^{(4)}(0)=0, p=0$ and the sum (77) reads, $\frac{\phi(0)}{\pi}=\operatorname{sgn} \mathcal{S}\left(\omega_{1}\right)=$ $\operatorname{sgn} \mathcal{S}\left(\omega^{*}\right)=1$, which accounts for the only positive root in the interval $\left.\omega \in\right] \frac{\pi}{\tau^{*}}, \frac{3 \pi}{2 \tau^{*}}[$. Note that in [21,54] the contour is taken in the right halfplane (i.e. $x_{0} \equiv 0$ ), which does not affect our result since integrals over $g_{2}$ and $g_{4}$ are vanishing. Hence,

$$
\lim _{R \rightarrow \infty} \frac{1}{2 \pi i} \int_{g_{3}} \frac{\partial_{s} \widetilde{Q}(s, \tau)}{\widetilde{Q}(s, \tau)} d s=1
$$

which is in agreement with (73) and (76).

Final step: In conclusion,

$$
\lim _{R \rightarrow \infty} \frac{1}{2 \pi i} \oint_{C_{R}} \frac{\partial_{s} \widetilde{Q}(s, \tau)}{\widetilde{Q}(s, \tau)} d s=0
$$

The last step concerns the value of the original (un-deflated) :

$$
\lim _{R \rightarrow \infty} \frac{1}{2 \pi i} \oint_{C_{R}} \frac{\partial_{s} Q(s, \tau)}{Q(s, \tau)} d s=\lim _{R \rightarrow \infty} \frac{1}{2 \pi i} \oint_{C_{R}} \frac{P^{\prime}(s)}{P(s)} d s
$$

where,

$$
\frac{1}{2 \pi i} \oint_{C_{R}} \frac{P^{\prime}(s)}{P(s)} d s=\frac{1}{2 \pi i} \int_{g_{1}} \frac{P^{\prime}(s)}{P(s)} d s+\frac{1}{2 \pi i} \int_{g_{2} \cup g_{4}} \frac{P^{\prime}(s)}{P(s)} d s+\frac{1}{2 \pi i} \int_{g_{3}} \frac{P(s)}{P(s)} d s .
$$

The first integral in the r.h.s. of (78) equals to 2 according to (66), the second integral equals 0 according to (70) and finally, the third integral over $g_{3}$ should be performed over the contour displayed in figure 12

$$
\begin{aligned}
\frac{1}{2 \pi i} \int_{g_{3}} \frac{P^{\prime}(s)}{P(s)} d s & =\frac{2}{\pi i} \int_{R}^{\epsilon} \frac{d \omega}{\omega}+\frac{2}{\pi} \int_{\frac{\pi}{2}}^{-\frac{\pi}{2}} d \theta+\frac{2}{\pi i} \int_{-\epsilon}^{-R} \frac{d \omega}{\omega} \\
& =\frac{2}{\pi i}(\ln \epsilon-\ln R)-2+\frac{2}{\pi i}(\ln (-R)-\ln (-\epsilon))=-2
\end{aligned}
$$

Therefore,

$$
\lim _{R \rightarrow \infty} \frac{1}{2 \pi i} \oint_{C_{R}} \frac{P^{\prime}(s)}{P(s)} d s=\lim _{R \rightarrow \infty} \frac{1}{2 \pi i} \oint_{C_{R}} \frac{\partial_{s} Q(s, \tau)}{Q(s, \tau)} d s=0 .
$$

The last result states that the deflation procedure of $[21,54]$ is equivalent to evaluating the integral over the small halfcircle of radius $\epsilon$ in figure 12. For an effective implementation in complex integral computations we refer the reader to [60]. 


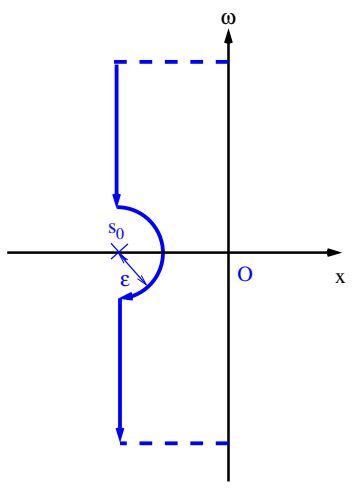

FIGURE 12. Part of the integration contour.

\section{REFERENCES}

[1] L. V. Ahlfors. Complex Analysis. McGraw-Hill, Inc., 1979.

[2] S. Amrane, F. Bedouhene, I. Boussaada, and S.-I. Niculescu. On qualitative properties of low-degree quasipolynomials: Further remarks on the spectral abscissa and rightmost-roots assignment. Bulletin Mathématique de la Société des Sciences Mathématiques de Roumanie, 61(109):361 $381,2018$.

[3] R. Bellman and K. Cooke. Differential-difference equations. Academic Press, New York, 1963.

[4] S.P. Bhattacharyya, H. Chapellat, and L.H. Keel. Robust Control: The Parametric Approach. Prentice-Hall information and system sciences series. Prentice Hall PTR, 1995.

[5] F.G Boese. Stability with respect to the delay: On a paper of k. 1. cooke and p. van den driessche. Journal of Mathematical Analysis and Applications, 228(2):293 - 321, 1998.

[6] I. Boussaada, I-C. Morarescu, and S-I. Niculescu. Inverted pendulum stabilization: Characterization of codimension-three triple zero bifurcation via multiple delayed proportional gains. Systems and Control Letters, 82:1 - 9, 2015.

[7] I. Boussaada and S-I. Niculescu. Computing the codimension of the singularity at the origin for delay systems: The missing link with Birkhoff incidence matrices. 21st International Symposium on Mathematical Theory of Networks and Systems, pages 1 - 8, 2014.

[8] I. Boussaada and S-I. Niculescu. Characterizing the codimension of zero singularities for time-delay systems. Acta Applicandae Mathematicae, 145(1):47-88, 2016

[9] I. Boussaada and S. I. Niculescu. Tracking the algebraic multiplicity of crossing imaginary roots for generic quasipolynomials: A Vandermondebased approach. IEEE Transactions on Automatic Control, 61:1601-1606, 2016.

[10] I. Boussaada and S-I. Niculescu. On the dominancy of multiple spectral values for time-delay systems with applications. IFAC-PapersOnLine, 51(14):55 - 60, 2018. 14th IFAC Workshop on Time Delay Systems TDS 2018.

[11] I. Boussaada, S-I. Niculescu, S. Tliba, and T. Vyhlídal. On the coalescence of spectral values and its effect on the stability of time-delay systems: Application to active vibration control. Procedia IUTAM, 22(Supplement C):75 - 82, 2017.

[12] I. Boussaada, S-I. Niculescu, and K. Trabelsi. Toward a decay rate assignment based design for time-delay systems with multiple spectral values. In Proceeding of the 23rd International Symposium on Mathematical Theory of Networks and Systems, pages 864-871, 2018.

[13] I. Boussaada, S. Tliba, S-I. Niculescu, H. Unal, and T. Vyhlídal. Further remarks on the effect of multiple spectral values on the dynamics of time-delay systems. application to the control of a mechanical system. Linear Algebra and its Applications, 542:589 - 604, 2018. Proceedings of the 20th ILAS Conference, Leuven, Belgium 2016.

[14] I. Boussaada, H. Unal, and S-I. Niculescu. Multiplicity and stable varieties of time-delay systems: A missing link. In Proceeding of the 22nd International Symposium on Mathematical Theory of Networks and Systems, pages 1-6, 2016.

[15] D. Breda. On characteristic roots and stability charts of delay differential equations. International Journal of Robust and Nonlinear Control, 22(8):892-917, 2012.

[16] K. L. Cooke. Stability analysis for a vector disease model. Rocky Mountain J. Math., 9:31-42, 1979.

[17] K-L. Cooke and P. van den Driessche. On zeroes of some transcendental equations. Funkcial. Ekvac., 29(1):77-90, 1986.

[18] K. Engelborghs and D. Roose. On stability of $1 \mathrm{~ms}$ methods and characteristic roots of delay differential equations. SIAM Journal on Numerical Analysis, 40(2):629-650, 2003.

[19] K. Gu, J. Chen, and V. Kharitonov. Stability of Time-Delay Systems. Birkhauser Boston, Inc., 2003.

[20] J. K. Hale and S. M. Verduyn Lunel. Introduction to functional differential equations, volume 99 of Applied Mathematics Sciences. Springer Verlag, New York, 1993.

[21] B.D. Hassard. Counting roots of the characteristic equation for linear delay-differential systems. Journal of Differential Equations, $136(2)$ :222 $235,1997$. 
[22] N. D. Hayes. Roots of the transcendental equation associated with a certain difference-differential equation. Journal of the London Mathematical Society, s1-25(3):226-232, 1950.

[23] T. Insperger and G. Stépán. Semi-Discretization for Time-Delay Systems: Stability and Engineering Applications. Applied Mathematics Sciences. Springer, 2011.

[24] V.L. Kharitonov, S-I. Niculescu, J. Moreno, and W. Michiels. Static output feedback stabilization: necessary conditions for multiple delay controllers. IEEE Trans. on Aut. Cont., 50(1):82-86, 2005.

[25] O. Kirillov and M. Overton. Robust stability at the swallowtail singularity. Frontiers in Physics, 1:24, 2013.

[26] M. Landry, S.A. Campbell, K. Morris, and C.O. Aguilar. Dynamics of an inverted pendulum with delayed feedback control. SIAM J. Appl. Dyn. Syst., 4(2):333-351, 2005.

[27] J.J. Loiseau. Invariant factors assignment for a class of time-delay systems. Kybernetika, 37(3):265 - 275, 2001.

[28] D. Ma and J. Chen. Delay margin of low-order systems achievable by pid controllers. IEEE Transactions on Automatic Control, 64(5):1958-1973, 2019.

[29] A. Manitius. Feedback controllers for a wind tunnel model involving a delay: Analytical design and numerical simulation. IEEE Transactions on Automatic Control, 29(12):1058-1068, 1984.

[30] A. Manitius and A. Olbrot. Finite spectrum assignment problem for systems with delays. IEEE Transactions on Automatic Control, 24(4):541-552, August 1979.

[31] M. Marden. Geometry of Polynomials. Number 3 in Geometry of Polynomials. American Mathematical Society, 1949.

[32] W. Michiels, I. Boussaada, and S-I. Niculescu. An explicit formula for the splitting of multiple eigenvalues for nonlinear eigenvalue problems and connections with the linearization for the delay eigenvalue problem. SIAM J. Matrix Analysis Applications, 38(2):599-620, 2017.

[33] W. Michiels, K. Engelborghs, P. Vansevenant, and D. Roose. Continuous pole placement for delay equations. Automatica, 38(5):747 - 761, 2002.

[34] W. Michiels and S-I. Niculescu. Stability and stabilization of time-delay systems, volume 12 of Advances in Design and Control. SIAM, 2007.

[35] A.V. Mikhailov. The methods of harmonie analysis in the theory of control. Avtomat. i Telemekh, (3):27-81, 1938.

[36] S. Mondie and J.J. Loiseau. Finite spectrum assignment for input delay systems. IFAC Proceedings Volumes, 34(23):201 - 206, 2001.

[37] T. Mori, N. Fukuma, and M. Kuwahara. On an estimate of the decay rate for stable linear delay systems. International Journal of Control, 36(1):9597, 1982.

[38] T. Mori and H. Kokame. Stability of $\dot{x}(t)=A x(t)+B x(t-\tau)$. IEEE Transactions on Automatic Control, 34:460-462, 1989.

[39] S-I. Niculescu and W. Michiels. Stabilizing a chain of integrators using multiple delays. IEEE Trans. on Aut. Cont., 49(5):802-807, 2004.

[40] S-I. Niculescu, W. Michiels, K. Gu, and C-T. Abdallah. Delay Effects on Output Feedback Control of Dynamical Systems, pages 63-84. Springer Berlin Heidelberg, Berlin, Heidelberg, 2010.

[41] H. Nyquist. Regeneration theory. The Bell System Technical Journal, 11(1):126-147, 1932.

[42] N. Olgac and R. Sipahi. An exact method for the stability analysis of time delayed linear time-invariant (lti) systems. IEEE Transactions on Automatic Control, 47(5):793-797, 2002.

[43] N. Olgac and R. Sipahi. An exact method for the stability analysis of time-delayed lti systems. IEEE Trans. Automat. Contr., 47:793-797, 2002.

[44] L. Pekař. Nyquist criterion for systems with distributed delays. Annals of DAAAM \& Proceedings, pages 485-487, 2011.

[45] L. Pekař and R. Prokop. Argument principle based stability conditions of a retarded quasipolynomial with two delays. In Proceedings of the 14th WSEAS International Conference on Systems: Part of the 14th WSEAS CSCC Multiconference - Volume I, ICS'10, pages 276-281. World Scientific and Engineering Academy and Society (WSEAS), 2010.

[46] G. Pólya and G. Szegó. Problems and Theorems in Analysis, Vol. I: Series, Integral Calculus, Theory of Functions. Springer-Verlag, New York, Heidelberg, and Berlin, 1972.

[47] A. Ramirez, S. Mondie, R. Garrido, and R. Sipahi. Design of proportional-integral-retarded (pir) controllers for second-order lti systems. IEEE Transactions on Automatic Control, (99):1-6, 2015.

[48] Z. V. Rekasius. A stability test for systems with delays. Joint Automatic Control Conference, 17:39, 1980.

[49] S. Ruan. Delay differential equations in single species dynamics. In Delay Differential Equations and Applications, pages 477-517. Springer, 2006.

[50] W. Rudin. Real and complex analysis. Mathematics series. McGraw-Hill, 1987.

[51] J. Sieber and B. Krauskopf. Bifurcation analysis of an inverted pendulum with delayed feedback control near a triple-zero eigenvalue singularity. Nonlinearity, 17:85-103, 2004.

[52] J. Sieber and B. Krauskopf. Extending the permissible control loop latency for the controlled inverted pendulum. Dynamical Systems, 20(2):189199, 2005.

[53] R. Sipahi, S. i. Niculescu, C. T. Abdallah, W. Michiels, and K. Gu. Stability and stabilization of systems with time delay. IEEE Control Systems, 31(1):38-65, Feb 2011.

[54] G. Stépán. Retarded Dynamical Systems: Stability and Characteristic Functions. Pitman research notes in mathematics series. Longman Scientific and Technical, 1989.

[55] I.H. Suh and Z. Bien. Proportional minus delay controller. IEEE Trans. on Aut. Cont., 24:370-372, 1979.

[56] J. Vanbiervliet, K. Verheyden, W. Michiels, and S. Vandewalle. A nonsmooth optimisation approach for the stabilisation of time-delay systems. ESAIM: COCV, 14(3):478-493, 2008.

[57] T. Vyhlídal and P. Zitek. Mapping based algorithm for large-scale computation of quasi-polynomial zeros. IEEE Transactions on Automatic Control, 54(1):171-177, 2009. 
[58] K. Walton and J. E. Marshall. Direct method for tds stability analysis. IEE Proceedings D - Control Theory and Applications, 134(2):101-107, 1987.

[59] E. M. Wright. Stability criteria and the real roots of a transcendental equation. Journal of the Society for Industrial and Applied Mathematics, 9(1):136-148, 1961.

[60] Q. Xu, G. Stépán, and Z. Wang. Delay-dependent stability analysis by using delay-independent integral evaluation. Automatica, 70:153 - 157, 2016.

[61] S. Yi, P. Nelson, and G. Ulsoy. Time-Delay Systems. WORLD SCIENTIFIC, 2010.

[62] P. Zitek, J. Fiser, and T. Vyhlidal. Dimensional analysis approach to dominant three-pole placement in delayed pid control loops. Journal of Process Control, 23(8):1063 - 1074, 2013. 